\title{
edoc
}

Institutional Repository of the University of Basel

University Library

Schoenbeinstrasse 18-20

CH-4056 Basel, Switzerland

http://edoc.unibas.ch/

Year: 2014

\section{Stable marriages and search frictions}

Lauermann, Stephan and Nöldeke, Georg

Posted at edoc, University of Basel

Official URL: http://edoc.unibas.ch/dok/A6243477

Originally published as:

Lauermann, Stephan and Nöldeke, Georg. (2014) Stable marriages and search frictions. Journal of economic theory, Vol. 151. S. 163-195.

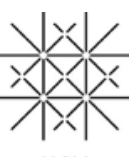




\title{
Stable Marriages and Search Frictions
}

\author{
Stephan Lauermann ${ }^{\mathrm{a}}$, Georg Nöldeke ${ }^{\mathrm{b}}$ \\ ${ }^{a}$ Department of Economics, University of Michigan, USA \\ ${ }^{b}$ Faculty of Business and Economics, University of Basel, Switzerland
}

\begin{abstract}
Stable matchings are the primary solution concept for two-sided matching markets with nontransferable utility. We investigate the strategic foundations of stability in a decentralized matching market. Towards this end, we embed the standard marriage markets in a search model with random meetings. We study the limit of steady-state equilibria as exogenous frictions vanish. The main result is that convergence of equilibrium matchings to stable matchings is guaranteed if and only if there is a unique stable matching in the underlying marriage market. Whenever there are multiple stable matchings, sequences of equilibrium matchings converging to unstable, inefficient matchings can be constructed. Thus, vanishing frictions do not guarantee the stability and efficiency of decentralized marriage markets.
\end{abstract}

JEL classification: C78, D83.

Keywords: Marriage Market, Nontransferable Utility, Stable Matchings, Search Frictions

\section{Introduction}

This paper considers a two-sided matching market with nontransferable utility: agents on each side of the market match with at most one agent from the other side of the market, and agents cannot make transfers. Following standard practice, we refer to such a market as a marriage market and use the corresponding terminology; see Roth and Sotomayor [26]. The central theoretical problem in a marriage market is to determine who will match with whom. The concept of stable matchings introduced in Gale and Shapley [17] provides an answer: a matching is stable if no matched agent prefers to be single and no pair of agents prefers each other to their assigned partner in the matching.

Most of the recent literature on matching considers questions of economic design in centralized markets. However, many of those markets that are analyzed using stability concepts, including the marriage market in its literal sense, are decentralized. Roth and Sotomayor [26, p.22] conjecture that even in a decentralized market, we might expect matchings to be stable if frictions are negligible in the sense that "the agents have a very good idea of one another's preferences and have easy access to each other." The purpose of this paper is to test this conjecture. In particular, we assume complete information about preferences and ask whether "easy access" to potential partners implies the stability of matchings.

Email addresses: slauerma@umich.edu (Stephan Lauermann), georg.noeldeke@unibas. ch (Georg Nöldeke) Accepted for Publication in The Journal of Economic Theory 
To investigate this question, we embed a marriage market in a search model with random meetings and investigate the limit of steady-state equilibria in the search model when search frictions vanish. Our approach is thus akin to the one in the literature on convergence to competitive equilibria in dynamic matching and bargaining games, surveyed in Osborne and Rubinstein [24] and Gale [16]. ${ }^{1}$ The concern motivating our paper is the same as the one motivating this literature, namely, to investigate the strategic foundations of stable outcomes in decentralized markets. The key difference is that we consider the nontransferable utility case.

In the underlying marriage market that we consider, there are a finite number of men and women. All individuals have strict preferences over mates and staying single; no further restrictions on preferences are imposed. In the search model there are continua of men and women with each individual in the marriage market represented by one of a finite number of types. The rate at which men and women meet one another is determined by the size of the population of agents searching for a potential partner according to a continuous contact function. If a man and a woman meet, they decide whether to accept each other. If both accept, the agents leave as a matched pair. Otherwise, both continue searching. The opportunity cost of rejecting a partner and waiting for a better match is an exogenous risk that an agent will have to abandon the search and remain single. Exogenously arriving unmatched men and women keep the stock of agents who are searching for partners from depleting. We study the matchings that result from steady-state equilibria and refer to these as equilibrium matchings.

Due to the randomness inherent in the contact process and the risk of exogenous exit, different agents of the same type will obtain different outcomes in a steady-state equilibrium. The equilibrium matchings arising in the search model thus correspond to fractional matchings as considered in Roth et al. [28]. We find it convenient to refer to matchings in which all agents of the same type receive the same match as simple matchings and to reserve the term fractional matchings for matchings that are not simple; the term matching encompasses both possibilities. The distinction between simple and fractional matching patterns plays a key role in our analysis.

In the search model the speed of the contact process determines how difficult it is to access potential partners and will thus serve as our measure of frictions. To investigate whether equilibrium matchings approximate stable matchings when search frictions are small, we study those matchings in the marriage market that can be obtained as limits of equilibrium matchings in the search model as the speed of the contact process goes to infinity. We refer to these matchings (both, simple and fractional) as limit matchings.

Our first main result, Proposition 1, is that a simple matching is a limit matching if and only if it is stable. At first glance, this result may seem to settle the question motivating this paper in the positive. It does not. The reason is that Proposition 1 does not preclude the existence of fractional limit matchings. To obtain firm conclusions about the stability of limit matchings, we need to address the existence of fractional limit matchings and their properties. Our second main result, Proposition 2, shows that fractional limit matchings exist if and only if there is more than one stable matching in the underlying marriage market. Fractional limit matchings have undesirable properties. We show that fractional limit matchings fail to satisfy a weak stability requirement that is implied by all existing stability notions for fractional matchings. In addition, fractional limit matchings are inefficient.

\footnotetext{
${ }^{1}$ A rather different approach to the emergence of stable matchings in marriage markets with decentralized decision making is pursued by Roth and Vande Vate [27]. They demonstrate that starting from any initial matching, the process of allowing randomly chosen "blocking pairs" of myopic agents to match converges to a stable matching. Their model abstracts from the strategic considerations and frictions that are at the heart of our approach.
} 
Taken together, our results identify a dichotomy between those marriage markets that have a unique stable matching and those that do not. In the first case, all equilibrium matchings converge to the unique stable matching when frictions vanish. In the second case, vanishing frictions do not imply stability. ${ }^{2}$

Our analysis is driven by two characterization results. The first of these, given in Lemma 7, provides necessary and sufficient conditions for a matching to arise as the equilibrium outcome in the search model. Theorem 1 builds on Lemma 7 to obtain a concise characterization of limit matchings in terms of the parameters of the underlying marriage market. These results not only yield Propositions 1 and 2, but also provide more general insights into the structure of equilibria with search frictions. We demonstrate this in Section 5 by obtaining counterparts to our limit results for the case of small search frictions.

Three key features differentiate our model and results from related contributions investigating the limits of steady-state equilibria in search models for vanishing frictions.

First, as we have already noted above, the literature on convergence to competitive equilibria in dynamic matching and bargaining games considers transferable utility, whereas we assume utility to be nontransferable. This difference is critical to our analysis. The reason is that with nontransferable utility agents may disagree about whether or not to form a match. ${ }^{3}$ For example, if a woman strictly prefers to match with the man she has met and the man is indifferent as to either accepting the match or continuing to search, there is no inducement the woman can offer to the man to break his indifference. ${ }^{4}$ Such disagreements play, as we demonstrate in Corollary 4 , an essential role in our construction of fractional limit matchings.

Second, the composition and size of the stock of searching agents are endogenously determined by agents' acceptance decisions and, in addition, depend on the speed of the contact process. Consequently, whether an agent has "easy access" to attractive partners depends not only on the search frictions per se, but also on the equilibrium behavior of all other agents and the properties of the contact function. Taking these effects into account differentiates our work from the two existing papers, namely Eeckhout [13] and Adachi [1], that investigate convergence to stable matchings in frictional search models with nontransferable utility: Adachi [1] assumes that the distribution of agents searching for a partner is exogenous, whereas Eeckhout [13] considers the special case of a market in which identical measures of men and women meet each other according to a contact function with constant returns to scale, thus eliminating many of the complications we encounter in our more general setting. Indeed, dealing with the endogeneity of the stock is one of the main challenges in proving our characterization of limit matchings. We believe that taking these effects into account is not only of technical but also of economic interest. $^{5}$

\footnotetext{
${ }^{2}$ A similar dichotomy has been observed in related problems. Sönmez [33] studies a general class of indivisible goods allocation problems, including the marriage market investigated here as a special case, and finds that there exists a Pareto efficient, individual rational, and strategy-proof solution only if the core correspondence is "essentially single-valued." Bloch and Diamantoudi [3] study a non-cooperative game of coalition formation and identify conditions which imply that all equilibria are efficient (in the sense of no-delay) and generate stable outcomes. In a marriage market these conditions are satisfied if the market itself and any "submarket" have a unique stable matching.

${ }^{3}$ In contrast, if transfers are possible, agents always agree whether a match should be formed, because the available surplus from the match is either positive or not. Smith [31] uses the observation that disagreements about matchings in social settings are common as an argument for the nontransferable utility model.

${ }^{4}$ Such indifferences may arise in equilibrium despite our assumption that preferences are strict because continuation payoffs are determined endogenously to equilibrate the market.

${ }^{5}$ Burdett and Coles [6] and Shimer and Smith [30] discuss the importance of the feedback effect from agents' decision
} 
Third, we consider the general version of the marriage model as introduced in Gale and Shapley [17], in which multiplicity of stable matchings is a common occurrence. This is in contrast to most of the literature studying search models with nontransferable utility which follows Becker [2] in assuming that agents agree on the ranking of their potential partners. ${ }^{6}$ In such models with vertical heterogeneity, there is a unique stable matching featuring positive assortative matching. ${ }^{7}$ Our analysis not only shows that in models with vertical heterogeneity the convergence to a stable outcome is assured, but also demonstrates that this result is not robust. Clearly, to reach the latter conclusion, it is essential to consider more general preference structures.

The papers most closely related to ours are Eeckhout [13] and Adachi [1]. These authors also consider the relationship between stable matchings in a marriage market and the equilibrium matchings in a search model when frictions become negligible. Both papers find convergence to stable matchings. This is consistent with our findings: The underlying marriage market in Eeckhout [13] features vertical heterogeneity and thus has a unique stable matching. Adachi [1], who considers general preferences as we do, eliminates fractional limit matchings from consideration by requiring an agent to accept a partner whenever the agent is indifferent as to accepting the partner or continuing to search.

Section 2 introduces the marriage market model, discusses stable matchings, and introduces the notions of coherent and regret-free matchings that play a key role in our analysis. Section 3 embeds the marriage market in a search model, defines equilibrium matchings, and notes some of their properties. We present our limit results for the case of vanishing frictions in Section 4. As a byproduct of our convergence analysis, we obtain results for search with small frictions. These are noted in Section 5. Section 6 concludes.

\section{Marriage Market}

\subsection{Agents and Preferences}

There are two finite, disjoint sets of agents: the set $M$ of men and the set $W$ of women. Each agent has a complete, transitive, and strict preference ordering over the set of agents on the other side of the market and the prospect of remaining single. We assume that the extension of this preference to the set of corresponding lotteries has an expected utility representation. We represent these preferences for a man $m \in M$ by the utilities $u(m, h) \in \mathbb{R}$, where $h \in W \cup\{m\}$, and for a woman $w \in W$ by $v(h, w) \in \mathbb{R}$, where $h \in M \cup\{w\}$. We normalize the utility of staying single to zero, that is $u(m, m)=v(w, w)=0$ holds for all $m \in M$, resp. $w \in W$. As preferences are strict, we have $u(m, w) \neq 0$ and $v(m, w) \neq 0$ for all $(m, w) \in M \times W$ as well as $u(m, w) \neq u\left(m, w^{\prime}\right)$ and $v(m, w) \neq v\left(m^{\prime}, w\right)$ for all $(m, w) \in M \times W, w^{\prime} \neq w \in W$, and $m^{\prime} \neq m \in M$. To avoid trivialities, we assume that there is at least one pair of agents $(m, w)$ such that $(m, w)$ find each other mutually acceptable, that is, $u(m, w)>0$ and $v(m, w)>0$ holds.

We refer to a tuple $(M, W ; u, v)$ satisfying the above assumptions as a marriage market.

to the steady-state distribution of types. In the context of the literature on convergence to competitive equilibria, Gale [15] has pioneered the investigation of models in which the stock of searching agents is endogenously determined and offers extensive discussion. See Lauermann [20] for further discussion.

${ }^{6}$ Burdett and Coles [7] and Smith [32] survey the literature. Notable contributions include McNamara and Collins [21], Johnstone et al. [18], Burdett and Coles [6], Morgan [23], Eeckhout [13], Bloch and Ryder [4], Smith [31]. Burdett and Wright [9] and Burdett et al. [8] also study search models with nontransferable utility, but consider a model with match-specific random shocks in which all agents on the same side of the market are identical ex-ante.

${ }^{7}$ See Eeckhout [14] and Clark [10] for more general conditions ensuring uniqueness of stable matchings. 


\subsection{Matchings and Payoffs}

A matching is given by an assignment matrix $x \in \mathbb{R}^{|M| \times|W|}$ satisfying

$$
\begin{aligned}
\sum_{w \in W} x(m, w) & \leq 1 \text { for all } m \in M, \\
\sum_{m \in M} x(m, w) & \leq 1 \text { for all } w \in W, \\
x(m, w) & \geq 0 \text { for all }(m, w) \in M \times W .
\end{aligned}
$$

If the assignment matrix in the above definition of a matching satisfies $x(m, w) \in\{0,1\}$ for all $(m, w) \in M \times W$, we say that the matching is simple; otherwise, it is fractional.

The interpretation of a simple matching is straightforward: $x(m, w)=1$ indicates that man $m$ and woman $w$ are matched, whereas $x(m, w)=0$ indicates that they are not. The constraints in (1) - (3) ensure that no individual has more than one partner in a simple matching. Simple matchings can also be described (Roth and Sotomayor [26, Definition 2.1]) by a matching function $\mu$ : $M \cup W \rightarrow M \cup W$ satisfying $\mu(m) \in W \cup\{m\}, \mu(w) \in M \cup\{w\}$, and $\mu^{2}(h)=h$ for all $m \in M$, $w \in W$, and $h \in M \cup W$. For every matching function having these properties, setting

$$
x(m, w)=1 \Leftrightarrow \mu(m)=w \Leftrightarrow \mu(w)=m
$$

produces the unique assignment matrix describing the corresponding simple matching (and vice versa).

The interpretation of fractional matchings ${ }^{8}$ depends on the context. One possibility is to view fractional matchings as describing lotteries over simple matchings. ${ }^{9}$ In this interpretation, $x(m, w)$ is the probability that a match between man $m$ and woman $w$ is formed, $x(m, m):=$ $1-\sum_{w \in W} x(m, w)$ is the probability that man $m$ remains single, and $x(w, w):=1-\sum_{m \in M} x(m, w)$ is the probability that woman $w$ stays single. Given any matching $x$, we say that $h \in M \cup W$ is fully matched if $x(h, h)=0$, partially matched if $0<x(h, h)<1$, and unmatched if $x(h, h)=1$ holds. Types $(m, w) \in M \times W$ are partners if $x(m, w)>0$ holds.

With every matching $x$, we associate the payoff vectors $U(x) \in \mathbb{R}^{|M|}$ and $V(x) \in \mathbb{R}^{|W|}$ given by

$$
\begin{aligned}
& U(m ; x)=\sum_{w \in W} x(m, w) u(m, w), \\
& V(w ; x)=\sum_{m \in M} x(m, w) v(m, w) .
\end{aligned}
$$

Recall that we have normalized the utility from staying single to zero. Hence, these payoffs correspond to the expected utilities of the matching $x$.

\footnotetext{
${ }^{8}$ Fractional matchings are studied (among others) by Vande Vate [35], Rothblum [29], Roth et al. [28], Kesten and Ünver [19], Echenique et al. [12]

${ }^{9}$ The Birkhoff-von Neumann theorem implies that every such lottery can be represented by an assignment matrix satisfying (1) - (3) and every such assignment matrix corresponds to a lottery over simple matchings. See Budish et al. [5] for an extensive discussion in the context of assignment problems.
} 


\subsection{Individual Rationality and Pairwise Stability}

A matching $x$ is individually rational if

$$
x(m, w)>0 \Rightarrow u(m, w) \geq 0 \text { and } v(m, w) \geq 0
$$

holds and it is pairwise stable if

$$
\text { there is no }(m, w) \in M \times W \text { s.t. } u(m, w)>U(m ; x) \text { and } v(m, w)>V(w ; x) \text {. }
$$

For simple matchings, we can use (4) to rephrase individual rationality and pairwise stability in terms of the matching function describing the simple matching. For simple matchings we have $U(m ; x)=u(m, \mu(m))$ and $V(w ; x)=v(\mu(w), w)$, so that such a matching is individually rational if and only if $u(m, \mu(m)) \geq 0$ holds for all $m$ and $v(\mu(w), w) \geq 0$ holds for all $w$. It is pairwise stable if and only if there does not exist $(m, w) \in M \times W$ such that $u(m, w)>u(m, \mu(m))$ and $v(m, w)>v(\mu(w), w)$ holds. These are the requirements that the simple matching is not blocked by any individual (individual rationality) or any pair of agents (pairwise stability), cf. Roth and Sotomayor [26, p. 21].

Under the lottery interpretation of fractional matchings, (7) corresponds to an ex-post individual rationality requirement. In contrast, (8) means that there is no blocking pair at the ex-ante stage before the outcome of the lottery (that is, a simple matching) is realized. While other formulations of individual rationality and pairwise stability for fractional matchings are possible, conditions (7) - (8) turn out to be the ones most relevant for our subsequent analysis.

\subsection{Stable Matchings}

A simple matching is stable if it is individually rational and pairwise stable. We recall the fact that every marriage market has a stable simple matching (Gale and Shapley [17]). Furthermore, our assumption that there is a mutually acceptable pair (that is, a pair satisfying $u(m, w)>0$ and $v(m, w)>0)$ implies that in every stable simple matching there exists $(m, w) \in M \times W$ such that $x(m, w)=1$ holds. (Equivalently, $\mu(h) \neq h$ holds for some $h \in M \cup W$.)

We extend stability to fractional matchings following Vande Vate [35] and say that a matching $x$ is stable if and only if there exist stable simple matchings $x^{1}, \ldots, x^{k}$ such that $x$ is a convex combination of $x^{1}, \ldots, x^{k} \cdot{ }^{10}$ Under the lottery interpretation of fractional matchings offered above, this means that stable fractional matchings can be obtained as lotteries over stable simple matchings.

Since the set of agents who are unmatched is the same for all stable simple matchings (see Roth and Sotomayor [26, Theorem 2.22]) and stable matchings, by definition, are convex combinations of stable simple matchings, every stable matching satisfies $x(h, h) \in\{0,1\}$ for all $h \in M \cup W$. Recalling our definition of a partially matched agent, we have the following result, which we record as a lemma for later reference.

Lemma 1. Let $x$ be a stable matching. Then there are no partially matched agents in $x$.

As alternative stability notions for fractional matchings have been proposed in the literature, ${ }^{11}$ we observe that for fractional matchings Lemma 1 is the only implication of stability that we use in our subsequent analysis.

\footnotetext{
${ }^{10}$ See Roth et al. [28] for the equivalence between this definition and the one given in Vande Vate [35].

${ }^{11}$ The stability notion by Vande Vate [35] used here has a natural interpretation as an ex-post stability requirement. From an ex-ante perspective, the notion of a strongly stable matching introduced in Roth et al. [28] may be more appealing. Our results continue to hold when using this alternative stability definition.
} 


\subsection{Coherent and Regret-Free Matchings}

The definitions and results in this subsection are not standard, but essential for our characterization of limit matchings in Section 4.

Definition 1. A matching is coherent if it is individually rational and pairwise stable, i.e., (7) (8) hold. A matching is regret-free if

$$
x(m, w)>0 \Rightarrow u(m, w) \geq U(m ; x) \text { and } v(m, w) \geq V(m ; x) .
$$

A simple matching is, by definition, coherent if and only if it is stable. For fractional matchings, this is not the case. ${ }^{12}$ Under the lottery interpretation of fractional matchings, regretfreeness can be understood as the requirement that any pair of agents who are matched with a strictly positive probability in $x$ weakly prefer to match with each other, rather than returning to the ex-ante stage before the outcome of the lottery is realized.

Every simple matching is regret-free because the two inequalities in (9) hold as equalities if $x(m, w)=1$. For fractional matchings, however, condition (9) has substantial bite.

Lemma 2. Let $x$ be a regret-free matching. Then every fully matched agent has a unique partner. In particular, $0<x(m, w)<1$ implies that $m$ and $w$ are partially matched.

Proof. Suppose $x$ satisfies (9). Assume $m \in M$ is fully matched. Using $x(m, m)=0$ and (5) we have

$$
\sum_{w \in W} x(m, w)[u(m, w)-U(m ; x)]=0 .
$$

Because $x$ satisfies (9), all the summands on the left side of this equality are positive. Hence, all summands are equal to zero. Consequently, $x(m, w)=0$ holds for all $w$ satisfying $u(m, w)-$ $U(m ; x) \neq 0$. Because preferences are strict, it follows that there is $w \in W$ satisfying $x(m, w)=1$. Hence, $m$ has a unique partner. An analogous argument shows that every fully matched $w \in W$ has a unique partner. Consequently, $0<x(m, w)<1$ implies $0<x(m, m)<1$ as well as $0<x(w, w)<1$ (because neither $m$ nor $w$ can be unmatched or fully matched if $0<x(m, w)<1$ holds).

The intuition behind Lemma 2 is simple: if every partner of an agent provides a payoff at least as high as the average payoff an agent receives, then it must either be the case that the agent only has one partner or it must be the case that there is a strictly positive probability that the agent remains unmatched. After all, in contrast to the children in Lake Wobegon, not all partners can be better than average.

As a straightforward implication of Lemmas 1 and 2, we obtain the result that stability and regret-freeness are incompatible for fractional matchings.

Lemma 3. Let $x$ be a regret-free fractional matching. Then $x$ is unstable.

Proof. Let $x$ be a regret-free fractional matching. By the definition of a fractional matching, there exists a pair $(m, w) \in M \times W$ such that $0<x(m, w)<1$ holds. Lemma 2 then implies that $m$ and $w$ are partially matched in $x$. By Lemma 1 , this implies that $x$ is unstable.

\footnotetext{
${ }^{12}$ This point is illustrated by the example below.
} 
The problem with regret-free fractional matchings is deeper than the instability identified in Lemma 3. Let us say that a matching is strongly inefficient if there exists a pair $(m, w) \in M \times W$ satisfying

$$
u(m, w)>0, v(m, w)>0, x(m, m)>0 \text {, and } x(w, w)>0 .
$$

In words, (10) means that both $m$ and $w$ stay single with strictly positive probability even though man $m$ and woman $w$ find each other acceptable. Hence, by the simple device of increasing $x(m, w)$ to $x^{\prime}(m, w)=x(m, w)+\min \{x(m, m), x(w, w)\}$ the payoff of both $m$ and $w$ can be strictly increased (without affecting the payoff of any other agent), thus generating a Pareto improvement.

Lemma 4. Let $x$ be a regret-free and individually rational fractional matching. Then $x$ is strongly inefficient.

Proof. Let $x$ be a regret-free and individually rational fractional matching. As in the proof of Lemma 3, we obtain the existence of a pair $(m, w)$ such that $0<x(m, w)<1, x(m, m)>0$, and $x(w, w)>0$ holds. Because $x$ is individually rational, the inequality $x(m, w)>0$ implies $u(m, w)>0$ and $v(m, w)>0$. Hence, $x$ is strongly inefficient.

We illustrate the above definitions and results with a simple example.

Example. Consider a marriage market with two men, $M=\left\{m_{1}, m_{2}\right\}$, and two women, $W=$ $\left\{w_{1}, w_{2}\right\}$. Preferences are described by the bi-matrix

$$
\begin{array}{l|c|c|} 
& \multicolumn{1}{c}{w_{1}} & \multicolumn{1}{c}{w_{2}} \\
\cline { 2 - 3 } m_{1} & 2,1 & 1,2 \\
\cline { 2 - 3 } m_{2} & 1,2 & 2,1 \\
\cline { 2 - 3 } & &
\end{array}
$$

where the first entry in the cell corresponding to $\left(m_{i}, w_{j}\right)$ is the payoff $u\left(m_{i}, w_{j}\right)$ and the second entry is the payoff $v\left(m_{i}, w_{j}\right)$. In this example, all matchings are individually rational. There are two stable simple matchings given by the assignment matrices

$$
x_{M}=\left[\begin{array}{ll}
1 & 0 \\
0 & 1
\end{array}\right] \text { and } x_{W}=\left[\begin{array}{ll}
0 & 1 \\
1 & 0
\end{array}\right] \text {. }
$$

The matching $x_{M}$ is the men-optimal matching, whereas the matching $x_{W}$ is the women-optimal matching; see Roth and Sotomayor [26, Definition 2.11].

There is one additional coherent and regret-free matching, namely the fractional matching

$$
x_{*}=\left[\begin{array}{ll}
\frac{1}{3} & \frac{1}{3} \\
\frac{1}{3} & \frac{1}{3}
\end{array}\right],
$$

resulting in the payoffs $U\left(m_{i} ; x_{*}\right)=V\left(w_{j} ; x_{*}\right)=1$ for $i, j=1,2$.

As implied by Lemma $3, x_{*}$ is unstable as each agent stays unmatched with probability $1 / 3$, whereas in a stable matching all agents are matched for sure. As implied by Lemma $4, x_{*}$ is strongly inefficient; a Pareto improvement would obtain from switching to the stable fractional matching

$$
x^{\prime}=\left[\begin{array}{ll}
\frac{1}{2} & \frac{1}{2} \\
\frac{1}{2} & \frac{1}{2}
\end{array}\right] \text {. }
$$




\section{Search}

\subsection{Search Process and Steady States}

We embed a marriage market $(M, W ; u, v)$ in a continuous-time search model with a continuum of agents, similar to the search model studied by Burdett and Coles [6]. To do so, we re-interpret the sets $M$ and $W$ as the set of possible types for men and women, respectively. For each $m \in M$ and $w \in W$, new agents of the corresponding type are born at a constant flow rate that is equal to $\eta>0$. Newborn agents start searching for partners and continue doing so until they either match with an agent of the opposite sex or exit the search process without having found a partner. Agents exit as unmatched singles at an exogenous rate $\delta>0 .{ }^{13}$ On the individual level, the exit rate acts like a discount rate and is a convenient way to model the opportunity costs of waiting for a better match. We thus do not consider any additional, explicit time discounting and assume the payoff of an agent of type $h$ who exits the search process in a partnership with another agent (or single) is the one from the underlying marriage market.

Let $f(m)>0$ denote the mass of men of type $m$ searching for a partner and let $f$ denote the corresponding vector of masses. Define $\bar{f}=\sum_{m \in M} f(m)$. Similarly, let $g(w)>0$ denote the mass of women of type $w$ searching for a partner, let $g$ be the corresponding vector, and define $\bar{g}=\sum_{w \in W} g(w)$. We suppress time indices because we consider steady states. The mass of meetings between men and women that occur per unit time is given by $\lambda \cdot C(\bar{f}, \bar{g})$. We refer to $C: \mathbb{R}_{+}^{2} \rightarrow \mathbb{R}_{+}$as the contact function and to the parameter $\lambda>0$ as the speed of the contact process. ${ }^{14}$ Only minimal assumptions are imposed on the contact function.

Assumption 1. The contact function $C$ is continuous and is strictly positive if and only if both of its arguments are strictly positive.

The contact functions commonly considered in the labor market search literature (Petrongolo and Pissarides [25]) satisfy Assumption 1, except for the linear matching function (Diamond and Maskin [11]), which violates the requirement that there are no meetings if the mass of men or women is zero; see Stevens [34].

We assume that the contact process is random in the sense that the fraction of meetings involving a man of type $m$ and a woman of type $w$ is given by $f(m) \cdot g(w) /(\bar{f} \cdot \bar{g})$. Defining $c: \mathbb{R}_{++}^{2} \rightarrow \mathbb{R}_{++}$by

$$
c(\bar{f}, \bar{g})=\frac{C(\bar{f}, \bar{g})}{\bar{f} \cdot \bar{g}},
$$

all men in the market thus meet women of type $w$ at rate

$$
\gamma(w ; f, g)=\lambda \cdot c(\bar{f}, \bar{g}) \cdot g(w)>0,
$$

and all women in the market meet men of type $m$ at rate

$$
\phi(m ; f, g)=\lambda \cdot c(\bar{f}, \bar{g}) \cdot f(m)>0 .
$$

\footnotetext{
${ }^{13}$ Our model of entry and exit is the one from Burdett and Coles [6]. As noted by Eeckhout [13], assuming that there is (i) a fixed population of infinitely lived agents who (ii) exit the search process only if they have found a partner and (iii) enter the search process when their partnership dissolves due to exogenous separation, yields identical steady-state conditions; see Shimer and Smith [30] and Smith [31].

${ }^{14}$ We refer to $C$ as a contact function rather than as a matching function because in our model not every meeting between agents needs to result in a match.
} 
If two agents meet, they observe each other's type and simultaneously decide whether to accept each other or not. If both accept, the agents leave the search process as a matched pair. If at least one agent rejects the match, both return to search. In line with our focus on steady states, we assume that all agents use time and history independent strategies. In addition, we restrict attention to strategy profiles that are symmetric in the sense that all agents of the same type use the same strategy. We may thus use a pair of functions $\beta_{M}: M \times W \rightarrow[0,1]$ and $\beta_{W}: M \times W \rightarrow[0,1]$ to describe a strategy profile, where $0 \leq \beta_{M}(m, w) \leq 1$ specifies the probability that a man of type $m$ accepts a match with a woman of type $w$ and, symmetrically, $0 \leq \beta_{W}(m, w)<1$ specifies the probability that a woman of type $w$ accepts a match with a man of type $m$. Let

$$
\alpha(m, w)=\beta_{M}(m, w) \cdot \beta_{W}(m, w) \in[0,1]
$$

denote the resulting probability that a meeting between a man of type $m$ and a woman of type $w$ results in a match. We use $\alpha \in[0,1]^{|M| \times|W|}$ to denote the corresponding matrix of acceptance probabilities.

Definition 2. A steady state is a tuple $(f, g, \alpha)$ satisfying

$$
\begin{aligned}
& \eta=f(m)\left[\delta+\sum_{w \in W} \alpha(m, w) \gamma(w ; f, g)\right] \text { for all } m \in M, \\
& \eta=g(w)\left[\delta+\sum_{m \in M} \alpha(m, w) \phi(m ; f, g)\right] \text { for all } w \in W .
\end{aligned}
$$

The left side of equations (15) - (16) represent the inflow of newborn agents of a given type. The right side is the corresponding outflow of single and matched agents. In a steady state these flows balance.

\subsection{Payoffs and Equilibria}

Given a steady state $(f, g, \alpha)$, the probability that a man of type $m$ is in a match with a woman of type $w$ when exiting from the search process is

$$
p_{(f, g, \alpha)}(m, w)=\frac{\alpha(m, w) \gamma(w ; f, g)}{\delta+\sum_{w^{\prime} \in W} \alpha\left(m, w^{\prime}\right) \gamma\left(w^{\prime} ; f, g\right)} .
$$

To see this, observe that $\delta+\sum_{w^{\prime} \in W} \alpha\left(m, w^{\prime}\right) \gamma\left(w^{\prime} ; f, g\right)$ is the exit rate of such a man, so that the expression on the right side of (17) is the probability of exiting in a match with $w$ conditional on exit. Similarly, the probability that a woman of type $w$ exits the search process in a partnership with a man of type $m$ is given by

$$
q_{(f, g, \alpha)}(m, w)=\frac{\alpha(m, w) \phi(m ; f, g)}{\delta+\sum_{m^{\prime} \in M} \alpha\left(m^{\prime}, w\right) \phi\left(m^{\prime} ; f, g\right)} .
$$

The expected payoffs associated with a steady state are therefore

$$
\begin{aligned}
U\left(m ; p_{(f, g, \alpha)}\right) & =\sum_{w \in W} p_{(f, g, \alpha)}(m, w) u(m, w), \\
V\left(w ; q_{(f, g, \alpha)}\right) & =\sum_{m \in M} q_{(f, g, \alpha)}(m, w) v(m, w) .
\end{aligned}
$$


An equilibrium is a steady state in which the acceptance probabilities $\alpha$ are consistent with the requirement that agents' acceptance decisions maximize their expected payoffs. Due to the stationarity of the environment, the continuation payoff of an agent who rejects a match and returns to the search process is as given in (19)- (20). Consequently, the optimality of strategies requires ${ }^{15}$

$$
\beta_{M}(m, w)= \begin{cases}0 & \text { if } u(m, w)<U\left(m ; p_{(f, g, \alpha)}\right) \\ 1 & \text { if } u(m, w)>U\left(m ; p_{(f, g, \alpha)}\right)\end{cases}
$$

and

$$
\beta_{W}(m, w)= \begin{cases}0 & \text { if } v(m, w)<V\left(w ; q_{(f, g, \alpha)}\right) \\ 1 & \text { if } v(m, w)>V\left(w ; q_{(f, g, \alpha)}\right)\end{cases}
$$

Observe that conditions (21) - (22) allow agents to randomize their acceptance decisions when indifferent as to whether to accept the current partner or continuing to search.

Conditions (21) - (22) are easily seen to imply that the acceptance probabilities $\alpha(m, w)$ as determined by (14) satisfy

$$
\alpha(m, w)= \begin{cases}0 & \text { if } u(m, w)<U\left(m ; p_{(f, g, \alpha)}\right) \text { or } v(m, w)<V\left(w ; q_{(f, g, \alpha)}\right), \\ 1 & \text { if } u(m, w)>U\left(m ; p_{(f, g, \alpha)}\right) \text { and } v(m, w)>V\left(w ; q_{(f, g, \alpha)}\right) .\end{cases}
$$

Vice versa, for any acceptance probabilities satisfying (23), there exist corresponding strategies satisfying (21) - (22). Just as we have done for steady states, we may thus define equilibria in terms of the stocks of agents searching for partners and the acceptance probabilities.

Definition 3. An equilibrium is a steady state $(f, g, \alpha)$ that satisfies condition $(23)$ for all $(m, w) \in$ $M \times W$.

\subsection{Steady-State Matchings and Equilibrium Matchings}

As shown in Lemma 5 below, the steady-state matching probabilities from the search model correspond directly to matchings in the underlying marriage market. Thus, even though the search model allows for the formation of inter-cohort partnerships and features a continuum of agents - whereas the underlying marriage market envisions a single cohort with a finite number of agents - there is a natural way to map equilibria of the search model into matchings. ${ }^{16}$

Definition 4. A steady state $(f, g, \alpha)$ induces the matching $x$, if the matching $x$ satisfies

$$
x=p_{(f, g, \alpha)}=q_{(p, f, \alpha)} .
$$

A matching $x$ is a steady-state matching if it is induced by some steady state $(f, g, \alpha)$; it is an equilibrium matching if it is induced by some equilibrium $(f, g, \alpha)$.

\footnotetext{
${ }^{15}$ We eliminate the dominated strategies in which an agent rejects a potential match that provides strictly higher utility than continued search.

${ }^{16}$ Such an interpretation of equilibrium outcomes relative to the static benchmark is conventional in the search literature: See, for example, Gale [15] and Lauermann [20].
} 
Condition (24) is the requirement that for all pairs of types $(m, w), x(m, w)$ is the probability that a man of type $m$ (resp. a woman of type $w$ ) will exit the search process in a match with a woman of type $w$ (resp. a man of type $m$ ). By (19) - (20) this condition ensures that in the steady state $(f, g, \alpha)$ every agent obtains matching probabilities and expected payoffs that are identical to the ones the agent obtains in the induced matching $x$ in the underlying marriage market.

The following lemma establishes a one-to-one relationship between steady states and steadystate matchings: every steady state induces a unique matching and every steady-state matching is induced by a unique steady state. To simplify notation, we let $\sigma_{x}=\sum_{m \in M} x(m, m)$ and $\tau_{x}=$ $\sum_{w \in W} x(w, w)$ for any matching $x$.

Lemma 5. Let $(f, g, \alpha)$ be a steady state. Then $x$ given by

$$
x(m, w)=\frac{\lambda}{\eta} \alpha(m, w) c(\bar{f}, \bar{g}) f(m) g(w) .
$$

is the unique matching induced by $(f, g, \alpha)$. Vice versa, if $x$ is a steady-state matching then $(f, g, \alpha)$ given by

$$
\begin{aligned}
f(m) & =\frac{\eta}{\delta} x(m, m), \\
g(w) & =\frac{\eta}{\delta} x(w, w), \\
\alpha(m, w) & =\frac{\delta^{2} x(m, w)}{\eta \lambda c\left(\frac{\eta}{\delta} \sigma_{x}, \frac{\eta}{\delta} \tau_{x}\right) x(m, m) x(w, w)},
\end{aligned}
$$

is the unique steady state inducing $x$.

Proof. See Appendix A.

It is immediate from equation (25) that a steady-state matching satisfies $x(m, w)>0$ if and only if the steady state inducing it satisfies $\alpha(m, w)>0$. Using the equilibrium requirement (23) it follows that every equilibrium matching is regret-free as defined by (9). As every agent has the option of rejecting all those potential partners generating a strictly negative payoff, it is also clear that equilibrium matchings are individually rational. We thus state without further proof:

Lemma 6. Every equilibrium matching is individually rational and regret-free.

The following lemma characterizes the set of all steady-state and equilibrium matchings in terms of the parameters $(M, W ; u, v)$ of the marriage market and the parameters $(\eta, \delta, \lambda, C)$ of the search model. Despite its simplicity, the result is important because it enables us to conduct the subsequent analysis without any explicit reference to the underlying steady states or equilibria. Instead, we can focus directly on the induced matchings and their properties. The lemma utilizes the one-to-one-relationship between steady states and steady-state matchings established in Lemma 5.

Lemma 7. A matching $x$ is a steady-state matching if and only if

$$
x(m, w) \leq \frac{\eta \lambda}{\delta^{2}} c\left(\frac{\eta}{\delta} \sigma_{x}, \frac{\eta}{\delta} \tau_{x}\right) x(m, m) x(w, w)
$$


holds for all $(m, w) \in M \times W$. A matching $x$ is an equilibrium matching if and only if, in addition,

$$
x(m, w)= \begin{cases}0 & \text { if } u(m, w)<U(m ; x) \text { or } v(m, w)<V(w ; x), \\ \frac{\lambda \eta}{\delta^{2}} c\left(\frac{\eta}{\delta} \sigma_{x}, \frac{\eta}{\delta} \tau_{x}\right) x(m, m) x(w, w) & \text { if } u(m, w)>U(m ; x) \text { and } v(m, w)>V(w ; x)\end{cases}
$$

holds for all $(m, w) \in M \times W$.

Proof. See Appendix A.

The characterization of steady-state matchings in Lemma 7 shows how the speed $\lambda$ of the contact process affects which matchings are feasible outcomes of the search process. In particular, because Assumption 1 ensures that the function $c$ is strictly positive on $\mathbb{R}_{++}$, it is immediate from (29) that every matching satisfying $x(h, h)>0$ for all $h \in M \cup W$ (that is, there are no fully matched agents) is a steady-state matching for sufficiently large $\lambda$. It follows that every matching can be obtained as the limit of a sequence of steady-state matchings as the speed of the contact process converges to infinity. This shows that the restriction to steady-state matchings does not cause any divergence between what is feasible in the underlying marriage market and what is feasible in the search model as the speed of the contact process goes to infinity. Consequently, the results that we describe in the following can be attributed to the properties of equilibrium matches, as given by the optimality conditions (30), rather than to some more superficial difference between the two settings we consider.

To illustrate the role of Lemma 7 and provide some intuition for our subsequent results, we return to the example introduced in Section 2.5.

Example (continued from Section 2.5). Consider acceptance probabilities of the form

$$
\alpha=\left[\begin{array}{ll}
1 & 0 \\
0 & 1
\end{array}\right]
$$

which result if men accept only their respective preferred partner types, while women accept all types. Lemma 5 implies that every steady-state matching that is induced by a steady state with these matching probabilities must be of the form

$$
x(s)=\left[\begin{array}{ll}
s & 0 \\
0 & s
\end{array}\right],
$$

with $0<s<1$. For every such $s$ there is a unique $\lambda(s)$ such that for $x(s)$, the inequality in (29) holds as an equality for the pairs $\left(m_{1}, w_{1}\right)$ and $\left(m_{2}, w_{2}\right)$. By Lemma 7, $x(s)$ is therefore a steadystate matching if and only if $\lambda \geq \lambda(s)$. Moreover, provided that $s \geq 1 / 2$, the matching $x(s)$ also satisfies (30). It then follows from Lemma 7 that $x(s)$ is an equilibrium matching for $\lambda=\lambda(s)$. It is not difficult to see from (29) that for $s \rightarrow 1, \lambda(s) \rightarrow \infty$. Thus, the men-optimal simple stable matching $x_{M}$ is the limit of equilibrium matchings as the speed of the contact process converges to infinity. Of course, an analogous construction can be used to show that there exists an equilibrium matching approximating the women-optimal matching $x_{W}$ for large contact speed.

Consider now the coherent and regret-free fractional matching

$$
x_{*}=\left[\begin{array}{cc}
\frac{1}{3} & \frac{1}{3} \\
\frac{1}{3} & \frac{1}{3}
\end{array}\right] \text {. }
$$

Because there is no fully matched agent in $x_{*}$, it follows from Lemma 7 that there exist $\underline{\lambda}$ such that for all $\lambda \geq \underline{\lambda}$ this matching is a steady-state matching (cf. the discussion after the statement of 
Lemma 7). Moreover, as $x_{*}$ is coherent and regret-free, (30) holds: because $x_{*}$ is regret-free, the first condition holds; because $x_{*}$ is pairwise stable, there is no pair of types for which the second set of inequalities applies. It follows from Lemma 7 that $x_{*}$ is an equilibrium matching whenever it is a steady-state matching, that is, whenever $\lambda \geq \underline{\lambda}$ holds. The key feature of the optimal strategy profile supporting $x_{*}$ as an equilibrium outcome is that every agent accepts his or her favorite partner with probability 1 and randomizes the acceptance decision in case the meeting is with the less desirable partner. This randomization is such that the probability of exiting the search process in a match with the less desirable partner is $1 / 3$. Such randomization is optimal because given $x_{*}$ each agent is indifferent towards accepting their less desirable partner.

\section{Matching with Vanishing Frictions}

In this section, we consider a fixed marriage market $(M, W ; u, v)$. We investigate the limit of equilibrium matchings as the search process becomes frictionless, in the sense that the speed of the contact process $\lambda$ converges to infinity. Throughout, the parameters $\delta>0, \eta>0$ and the contact function $C$ are kept fixed.

Definition 5. A matching $x_{*}$ is a limit matching if there exists a sequence $\left(\lambda_{k}\right)$ converging to infinity and a sequence of matchings $\left(x_{k}\right)$ converging to $x_{*}$ such that for all $k$ the matching $x_{k}$ is an equilibrium matching for the search model with parameters $\left(\eta, \delta, \lambda_{k}, C\right)$.

It is easy to see that the individual rationality and regret-freeness of equilibrium matchings (cf. Lemma 6) is preserved in the limit. The following theorem establishes that strengthening individual rationality to coherency yields a necessary and sufficient condition for a matching to be a limit matching. This result holds without any restrictions on the structure of the underlying marriage market and for all contact functions satisfying Assumption 1.

Theorem 1. A matching is a limit matching if and only if it is coherent and regret-free.

Proof. See Appendix B.

The intuition for the coherency of limit matchings is straightforward. Suppose that there exists a pair of types $(m, w)$ violating the pairwise stability condition (8) in the limit matching $x_{*}$. For sufficiently large $\lambda_{k}$, the optimality of equilibrium strategies then implies that every meeting between agents of types $m$ and $w$ results in a match. Provided that the rate at which such agents meet each other converges to infinity, this implies that the limit matching satisfies $x_{*}(m, w)=1$, contradicting the assumption that the pairwise stability condition is violated for these types.

The difficulty in making this intuition precise is that it is far from obvious that meeting rates converge to infinity as the speed of the matching technology converges to infinity. ${ }^{17}$ The problem is that an increase in the speed at which agents match (and thus exit from search) implies a reduction in the steady-state masses of agents, reducing the matching rates as given in (12) and (13). Furthermore, given the weak assumptions we have imposed on the contact function, such

\footnotetext{
${ }^{17}$ This is in contrast to a "cloning model", such as the one studied by Adachi [1]. In a cloning model, the steadystate masses $(f, g)$ are taken as given so that the population share of each type is strictly positive and constant along the sequence. Therefore, the matching rate necessarily converges to infinity as the speed of the contact process does so. Hence, in such a model, the counterpart to the necessary conditions for limit matchings in Theorem 1 is straightforward.
} 
a reduction in the steady-state stocks may reduce $c(\bar{f}, \bar{g})$, introducing a second effect countervailing the increase in the speed of the contact process. Taken together, these effects generate the possibility that the meeting rate for some type of agent may converge to a finite limit as $\lambda$ converges to infinity. Indeed, sequences of steady-state matchings with this property are easily constructed. The main subtlety in proving the coherency of limit matchings is to exclude this possibility for sequences of equilibrium matchings. In doing so, we exploit the continuity of the contact function imposed in Assumption 1 and make use of the characterization of regret-free matchings in Lemma 2.

The main challenge in proving the sufficiency of the conditions in Theorem 1 lies in showing that every coherent and regret-free fractional matching can be obtained as limit matching. In essence, given such a matching $x_{*}$, we must construct a sequence of equilibria such that, for every pair of types $(m, w)$ satisfying $0<x_{*}(m, w)<1$, the associated sequence of matching probabilities converges to an interior limit. This in turn requires that along the sequence at least one of the types $m$ and $w$ is indifferent between accepting the match with his or her designated partner, whereas the other type weakly prefers to accept the match.

As we have noted in Section 2.5, a simple matching is stable if and only if it is coherent. In addition, every simple matching is regret-free. Theorem 1 therefore implies:

Proposition 1. A simple matching is a limit matching if and only if it is stable.

Because stable simple matchings exist, Proposition 1 implies the existence of a simple limit matching as a by-product.

If one restricts attention to simple matchings, Proposition 1 indicates that the sets of limit matchings and stable matchings are identical. For fractional matchings, however, this equivalence breaks down. In fact, from the characterization of limit matchings in Theorem 1 and the results obtained in Lemmas 3 and 4, it is immediate that fractional limit matchings, should they exist, are unstable and strongly inefficient. This prompts our next result, which states a necessary and sufficient condition for the existence of fractional limit matchings.

Proposition 2. Fractional limit matchings exist if and only if there exists more than one stable simple matching.

Proof. See Appendix C.

The proof of Proposition 2 shows that a marriage market $(M, W ; u, v)$ possesses a regretfree and coherent fractional matching if and only if there exists more than one stable simple matching. To the best of our knowledge, this is a novel result. Combining it with Theorem 1 yields Proposition 2. To obtain the result that the existence of a regret-free and coherent fractional matching implies the existence of multiple simple matchings, we show that given any such fractional matching it is possible to construct two distinct stable simple matchings. These two simple matchings support the original fractional matching in the sense that the union of any agent's partners in the two simple matchings coincides with his or her set of partners in the fractional matching. Proving the other direction of the equivalence in the statement of Proposition 2 is harder and relies on the lattice-structure of the set of stable simple matchings; see Roth and Sotomayor [26, Chapter 3]. The difficulty is that not every pair of stable simple matchings supports a coherent and regret-free fractional matching. However, any two stable simple matchings, which have the property that all men have the same preferences over these two matchings and that there is no stable simple matching "between" those two in the men's preference ordering, support a coherent and regret-free fractional matching. In fact, our proof shows more, namely, 
that for every pair of such consecutive stable simple matchings there is exactly one coherent and regret-free fractional matching supported by this pair. The proof is then completed by the observation that consecutive stable simple matchings exist whenever there is more than one stable simple matching. In particular, if there are exactly two stable simple matchings in the marriage market, then - as illustrated by the example discussed in Sections 2.5 and 3.3 - there is a unique regret-free and coherent fractional matching and, thus, a unique fractional limit matching.

As an immediate consequence of the preceding results, we have the result advertised in the abstract.

Corollary 1. If there is a unique stable matching in the underlying marriage market, then this matching is the unique limit matching. If there are multiple stable matchings in the underlying marriage market, then there exist unstable and strongly inefficient limit matchings.

Proof. Suppose there is a unique stable matching, which then must be simple. From Proposition 1 this matching is a limit matchings and there are no other simple limit matchings. Because of Proposition 2, there are no fractional limit matching. Therefore, the unique stable simple matching is the unique limit matching.

Suppose that there is more than one stable matching and, thus, more than one stable simple matching. From Proposition 2, this implies that a fractional limit matching exists. From Theorem 1 , this fractional matching is coherent and regret-free. From Lemmas 3 and 4, it is unstable and strongly inefficient.

\section{Matching with Small Frictions}

Our analysis of the limit behavior of equilibrium matchings for vanishing frictions implies a number of results for the structure of equilibrium matchings with small frictions, that is, for sufficiently high but finite $\lambda$. We believe that some of these results may be of independent interest and thus present them here. Doing so should also help to clarify the structure of the proofs for our limit results. As in Section 4, we treat the parameters of the marriage market $(M, W ; u, v)$ and the parameters $(\eta, \delta, C)$ as given.

We first observe that in the process of proving Theorem 1 and Proposition 2 we have established a simple lower bound for the number of equilibria. In particular, for small frictions existence of equilibria is assured. ${ }^{18}$

Corollary 2. Let $n \geq 1$ denote the number of stable simple matchings in the marriage market. Then there exists $\underline{\lambda}_{e}>0$ such that for all $\lambda>\underline{\lambda}_{e}$ at least $2 n-1$ equilibria in the search model exist.

Proof. In a marriage market with $n$ stable simple matchings, there are at least $n-1$ distinct pairs of consecutive stable matchings (corresponding to those pairs connected by a branch of the lattice of stable matchings; cf. Roth and Sotomayor [26, Section 3.2]). Lemma 13 in Appendix $\mathrm{C}$ then implies that there are at least $n-1$ distinct coherent and regret-free fractional matchings. As every stable simple matching is coherent and regret-free it follows that there are at least $2 n-1$

\footnotetext{
${ }^{18}$ There are very few existence results for equilibria in search models with nontransferable utility in which the distribution of searching agents is endogenously determined, as it is in our model. In particular, Burdett and Coles [6] and Smith [31] establish existence for any level of frictions, but consider models with a unique stable matching in the underlying marriage market and impose stringent restrictions on the search technology.
} 
coherent and regret-free matchings. Label these matchings $x_{i}$ with $i=1, \ldots, 2 n-1$. Lemma 9 in Appendix $\mathrm{B}$ establishes that for each of the matchings $x_{i}$ there exists $\underline{\lambda}_{e_{i}}>0$ such that for any $\lambda>\underline{\lambda}_{e_{i}}$ an associated equilibrium matching, that we label $x_{i}^{\lambda}$, can be constructed such that these matchings satisfy $\lim _{\lambda \rightarrow \infty} x_{i}^{\lambda}=x_{i}$. As the matchings $x_{1}, \ldots, x_{2 n-1}$ are distinct, it follows that there exist $\underline{\lambda}_{e} \geq \max _{i} \underline{\lambda}_{e_{i}}$ such that $x_{i}^{\lambda} \neq x_{j}^{\lambda}$ holds for all $i \neq j$ and $\lambda>\underline{\lambda}_{e}$, establishing the existence of $2 n-1$ distinct equilibrium matchings for all such $\lambda$. As the relationship between equilibrium matchings and equilibria is one-to-one (cf. Definition 3 and Lemma 5), this implies the existence of $2 n-1$ distinct equilibria for $\lambda>\underline{\lambda}_{e}$.

The intuition why (for small frictions) there should be at least as many equilibria in the search model as there are stable simple matchings in the underlying marriage market is straightforward and captured by our discussion of the example in Section 3.3. ${ }^{19}$ Consider any given type $h$. If the agents of all other types only accept those matches involving a partner at least as attractive as the one assigned to them in a given stable simple matching $x$, then for small frictions agents of type $h$ will also find it optimal to follow the same rule. Given such a strategy profile and a corresponding steady state, we have an equilibrium in which only those meetings between pairs of agents for whom $x(m, w)=1$ holds, will form. ${ }^{20}$ The additional equilibria whose existence is asserted in Corollary 2 correspond to fractional limit matching.

From Corollary 2, uniqueness of stable matchings is a necessary condition for the uniqueness of equilibrium when frictions are small. The following result addresses the question whether this condition is also sufficient. We first demonstrate that the acceptance probabilities are uniquely determined for small frictions. This is intuitive: for sufficiently large $\lambda$, agents' expected payoffs must be close to their payoffs in the stable matching, and, for these payoffs, the only matches accepted by both agents in a meeting are those between types that are partners in the stable matching. Still, multiple steady-states may be compatible with the same acceptance behavior. This is because Assumption 1 imposes only very weak conditions on the contact function. Using the characterization of steady-state matchings in Lemma 7, we can exclude this possibility for the prominent example of a quadratic contact function as studied, for instance, in Shimer and Smith [30] and Smith [31]. ${ }^{21}$

Corollary 3. Suppose there is a unique stable matching $x_{*}$ in the marriage market. Then there exists $\underline{\lambda}_{q}>0$ such that for all $\lambda>\underline{\lambda}_{q}$ equilibrium acceptance probabilities are uniquely determined and given by $\alpha(m, w)=x_{*}(m, w) \in\{0,1\}$. If the contact function is quadratic,

$$
C(\bar{f}, \bar{g})=\bar{f} \cdot \bar{g},
$$

equilibrium is unique for $\lambda>\underline{\lambda}_{q}$.

\footnotetext{
${ }^{19}$ Just as we did in the discussion of the example, the following heuristic discussion takes for granted what we have established in the proof of Theorem 1, namely, that despite the endogeneity of the population small frictions imply "easy access" to potential partners (cf. the discussion following the statement of Theorem 1). It also takes for granted that for the strategy profile under consideration there exists a corresponding steady state when frictions are sufficiently small which is another implication of the proof of Theorem 1.

${ }^{20}$ This source of multiplicity is distinct from the source of multiplicity observed in Burdett and Coles [6, Example 3] who demonstrate that (for sufficiently high frictions) there may be multiple equilibria even when there is a unique stable matching. The multiplicity in Burdett and Coles is driven by the endogenous population of agents searching for a partner The multiplicity of equilibria we discuss here also arises in a model with a fixed population; see Adachi [1].

${ }^{21}$ While the structure of our argument is different, the role of the quadratic contact function in it is the same as in Shimer and Smith [30] and Smith [31], namely, to ensure that there is a unique steady state associated with a given strategy profile.
} 
Proof. See Appendix D.

We have noted in Section 2 that our equilibrium definition allows agents to randomize their acceptance decisions when indifferent and indicated in the Introduction that this assumption is important for our results. Our final result demonstrates that, as suggested by the discussion of our example in Section 3.3, such mixing plays no role in obtaining stable simple matchings as limit matchings, but is essential in constructing equilibria with associated matchings converging to fractional limit matchings.

Corollary 4. Given a sequence $\left(\lambda_{k}\right)$ converging to infinity, let $\left(f_{k}, g_{k}, \alpha_{k}\right)$ be a sequence of equilibria with induced steady-state matchings $x_{k}$ converging to a limit matching $x_{*}$. If $x_{*}$ is simple, then for all $k$ sufficiently large, $\alpha_{k}(m, w) \in\{0,1\}$ for all pairs of types $(m, w)$. If $x_{*}$ is fractional, then for all $k$ sufficiently large, there exists a pair of types $(m, w)$ such that $0<\alpha_{k}(m, w)<1$, $u(m, w)=U\left(m ; x_{k}\right)$, and $v(m, w)>V\left(w ; x_{k}\right)$.

Proof. For simple $x_{*}$ the result follows from the argument given in the first part of the proof of Corollary 3 leading up to Equations (D.3) and (D.4).

For every fractional limit matching $x_{*}$, the proof of Lemma 10 establishes the existence of a pair $(m, w)$ such that (a) $0<x_{*}(m, w)<1$, (b) $0<x_{*}(m, m)<1$, (c) $0<x_{*}(w, w)<1$, (d) $u(m, w)=U\left(m ; x_{*}\right)$ and, (e) $v(m, w)>V\left(w ; x_{*}\right)$; see the right-most parts of Equations (C.1) and (C.2). The continuity of the expected payoffs and the convergence of $x_{k}$ imply that for sufficiently large $k, v(m, w)>V\left(w ; x_{k}\right)$. Moreover, from Equation (28), the first three of these conditions imply that for every sequence of equilibria with induced matchings $\left(x_{k}\right)$ converging to $x_{*}$, for $k$ sufficiently large, the acceptance probability must satisfy $0<\alpha_{k}(m, w)<1$. This, $v(m, w)>V\left(w ; x_{k}\right)$, and the equilibrium condition (23) imply $u(m, w)=U\left(m ; x_{k}\right)$.

When combined with Proposition 1, Corollary 4 implies that a matching can be obtained as the limit of a sequence of "pure" equilibria if and only if it is a stable simple matching. Thus, we verify that the result by Adachi [1]—who restricts attention to strategy profiles in which agents accept when indifferent and obtains the set of stable simple matchings as the counterpart to our limit matchings - continues to hold when the stock is endogenous and, in addition, we identify the ruling out of mixed acceptance decisions as critical for such a conclusion. The second part of the conclusion in Corollary 4 implies further that a weaker restriction on agents' strategies, namely requiring indifferent agents to accept a match if the partner strictly prefers doing so, will also eliminate all fractional limit matchings. ${ }^{22}$

In our view, allowing for mixed acceptance decisions is natural. First, with an endogenous stock, simple examples demonstrate that mixed acceptance decisions are necessary to ensure equilibrium existence for all parameters. Second, the inability to offer an inducement to break the indifference of a partner is a central feature of the nontransferable utility setup. Strikingly, our analysis isolates precisely this feature as causing the existence of unstable and inefficient limit matchings.

\section{Concluding Remarks}

In this paper, we have revisited the classical marriage problem and its solution concept, namely, stability, in a frictional decentralized environment. We have demonstrated that when

${ }^{22} \mathrm{We}$ are grateful to an anonymous reviewer for noting this implication of our analysis. 
frictions vanish, the set of equilibrium matchings converges to the set of stable matchings if and only if there is a unique stable matching. Otherwise, additional, unstable and inefficient fractional matchings arise in the limit.

Many of the marriage markets considered in the economic literature possess a unique stable matching. It is thus an important class of marriage markets for which our Corollary 1 confirms the conjecture by Roth and Sotomayor [26] that stable matchings approximate equilibrium matchings in marriage markets with negligible frictions. However, our analysis reveals that the uniqueness of stable matchings plays an essential role in obtaining this conclusion.

\section{Acknowledgments}

We thank Gadi Barlevy, Francis Bloch, Jeremy Fox, Jacob Goeree, Alexey Kushnir, Konrad Mierendorff, Benny Moldovanu, Larry Samuelson, Lones Smith, Gabor Virag, and Randy Wright for helpful comments, as well as the editor and the anonymous reviewers for their suggestions. Isaac Sorkin and Qinggong Wu provided excellent research assistance.

\section{Appendix A. Proofs of Lemmas 5 and 7}

Proof of Lemma 5. Let $(f, g, \alpha)$ be a steady state and let $x$ satisfy (25). Clearly, every steady state induces at most one matching. Hence, to establish the first claim in the lemma, it suffices to show that $x$ satisfies (24) and the conditions for a matching, (1) - (3). From (15), we have

$$
\frac{1}{\eta} f(m)=\frac{1}{\delta+\sum_{w \in W} \alpha(m, w) \gamma(w ; f, g)} .
$$

Substituting this expression into (25), using the definition of $\gamma(w ; f, g)$ that is given in (12), and the definition of $p_{(f, g, \alpha)}$ from equation (17), establishes the equality between the first and the second expression in (24). An analogous calculation using (13), (16), and (18) establishes equality between the first and the third expression in (24). It is immediate from $(25)$ that $x(m, w) \geq 0$ holds for all pairs $(m, w)$. From (17) and (24) we have $\sum_{w \in W} x(m, w)=\sum_{w \in W} p_{(f, g, \alpha)}(m, w) \leq 1$. From (18) and (24), we have $\sum_{m \in M} x(m, w)=\sum_{m \in M} q_{(f, g, \alpha)}(m, w) \leq 1$.

Let $x$ be a steady-state matching and let $(f, g, \alpha)$ be a steady state inducing it. Summing (25) over all $w$ and using (12) and (15), we obtain

$$
x(m, m)=1-\sum_{w \in W} x(m, w)=1-f(m) \sum_{w \in W} \alpha(m, w) \gamma(w ; f, g) / \eta=\frac{\delta}{\eta} f(m)>0,
$$

implying (26). An analogous argument using (13) and (16) yields

$$
x(w, w)=1-\sum_{m \in M} x_{f, g, \alpha}(m, w)=1-g(w) \sum_{m \in M} \alpha(m, w) \phi(m ; f, g) / \eta=\frac{\delta}{\eta} g(w)>0,
$$

implying (27). To obtain (28), substitute from (26) - (27) into (25) and solve for $\alpha(m, w)$.

Proof of Lemma 7. Let $x$ be a steady-state matching. From Lemma 5, the steady-state $(f, g, \alpha)$ inducing $x$ satisfies (28). Since $(f, g, \alpha)$ is a steady-state, $\alpha$ satisfies $\alpha(m, w) \leq 1$ for all $(m, w)$, implying (29). 
Suppose $x$ is a matching satisfying (29) for all $(m, w) \in M \times W$. We show that there is a steady state, defined by (26) - (28), that induces $x$. First, (29) implies that $x(m, m)>0$ and $x(w, w)>0$ hold for all $m$ and $w .{ }^{23}$ Therefore, (26) - (27) define strictly positive stocks $(f, g)$. In addition, the acceptance probabilities $\alpha$ as given by (28) are well-defined and satisfy $0 \leq \alpha(m, w) \leq 1$ for all $(m, w)$. It remains to show that the tuple $(f, g, \alpha)$ defined this way is indeed a steady state and induces $x$. Using (26) - (28) we obtain

$$
\begin{aligned}
\frac{f(m)}{\eta}\left[\delta+\lambda c(\bar{f}, \bar{g}) \sum_{w \in W} \alpha(m, w) g(w)\right] & =x(m, m)+\sum_{w \in W} x(m, w), \\
\frac{g(w)}{\eta}\left[\delta+\lambda c(\bar{f}, \bar{g}) \sum_{m \in M} \alpha(m, w) f(m)\right] & =x(w, w)+\sum_{m \in M} x(m, w),
\end{aligned}
$$

for all $m \in M$ and $w \in W$. The right sides of these equations are equal to 1 . It follows that $(15)$ and (16) are satisfied. Hence, $(f, g, \alpha)$ is a steady state. Reversing the substitutions yielding Lemma 5, we have that $(f, g, \alpha)$ induces $x$. Hence, every matching satisfying (29) is a steady-state matching.

Equation (28) holds whenever a matching $x$ is induced by the steady state $(f, g, \alpha)$. Therefore, (23) implies that every equilibrium matching satisfies (30). Conversely, if a steady-state matching $x$ satisfies (30), then the steady state that induces it satisfies (23). Thus, a steady-state matching $x$ is an equilibrium matching if and only if it satisfies (30).

\section{Appendix B. Proof of Theorem 1}

Throughout the following proof, we set $\delta=1$ and $\eta=1$ to simplify notation. Doing so is without loss of generality because the effects of the parameters $\delta$ and $\eta$ may be subsumed in the contact function. More precisely, given any parameters $(\eta, \delta, \lambda, C)$ of the search model, there exists a contact function $\hat{C}$ such that a matching $x$ is a steady state (or equilibrium) matching for the parameters $(\eta, \delta, \lambda, C)$ if any only if it a steady state (or equilibrium) matching for the parameters $(1,1, \lambda, \hat{C}) .{ }^{24}$ We prove the Theorem using two lemmas.

Lemma 8. If $x_{*}$ is a limit matching, then it is coherent and regret-free.

Proof. Let $x_{*}$ be a limit matching, and let $\left(\lambda_{k}\right)$ and $\left(x_{k}\right)$ be the corresponding sequences satisfying the properties in Definition 5. For all $m \in M$ and $w \in W$ define the corresponding sequences of

\footnotetext{
${ }^{23}$ Suppose, for instance, $x(m, m)=0$. Then, (29) implies $x(m, w)=0$ for all $w$ and, thus, $x(m, m)=1-\sum_{w} x(m, w)=1$, a contradiction.

${ }^{24}$ For $(\sigma, \tau) \in \mathbb{R}_{+}^{2}$ define

$$
\hat{C}(\sigma, \tau)=\frac{1}{\eta} C\left(\frac{\eta}{\delta} \sigma, \frac{\eta}{\delta} \tau\right)
$$

and for $(\sigma, \tau) \in \mathbb{R}_{++}^{2}$ let $\hat{c}(\sigma, \tau)=\hat{C}(\sigma, \tau) /(\sigma \tau)$. Then,

$$
\frac{\eta}{\delta^{2}} c\left(\frac{\eta}{\delta} \sigma, \frac{\eta}{\delta} \tau\right)=\frac{1}{\eta} \frac{C\left(\frac{\eta}{\delta} \sigma, \frac{\eta}{\delta} \tau\right)}{\eta \sigma \tau}=\frac{\hat{C}(\sigma, \tau)}{\sigma \tau}=\hat{c}(\sigma, \tau)
$$

so that a matching $x$ satisfies (29), resp. (30), given the parameters $(\eta, \delta, \lambda, C)$ if and only if $x$ satisfies the same conditions given the parameters $(1,1, \lambda, \hat{C})$.
} 
meeting rates $\left(\phi_{k}(m)\right)$ and $\left(\gamma_{k}(w)\right)$ by ${ }^{25}$

$$
\begin{aligned}
\phi_{k}(m) & :=\lambda_{k} c\left(\sigma_{x_{k}}, \tau_{x_{k}}\right) x_{k}(m, m), \\
\gamma_{k}(w) & :=\lambda_{k} c\left(\sigma_{x_{k}}, \tau_{x_{k}}\right) x_{k}(w, w) .
\end{aligned}
$$

Throughout we assume that $\phi_{k}(m)$ and $\gamma_{k}(w)$ converge to $\phi_{*}(m) \in \mathbb{R} \cup\{\infty\}$, and $\gamma_{*}(w) \in \mathbb{R} \cup\{\infty\}$ for all $m$ and $w$, respectively. ${ }^{26}$

We first show that $x_{*}$ is individually rational and regret free. Suppose that $x_{*}(m, w)>0$ holds. As $x_{k}$ converges to $x_{*}$, there exists $K$, such that for all $k>K$ we have $x_{k}(m, w)>0$. As $x_{k}$ is an equilibrium matching, Lemma 6 implies $u(m, w) \geq 0$ and $v(m, w) \geq 0$. Therefore, $x_{*}$ is individually rational. Similarly, Lemma 6 implies $u(m, w) \geq U\left(m ; x_{k}\right)$ and $v(m, w) \geq V\left(w ; x_{k}\right)$ for all $k>K$. As $U(m ; \cdot)$ and $V(w, \cdot)$ are continuous in $x$, it follows that $u(m, w) \geq U\left(m ; x_{*}\right)$ and $v(m, w) \geq V\left(w ; x_{*}\right)$ hold. Hence, $x_{*}$ is regret-free.

It remains to show that $x_{*}$ is pairwise stable. Suppose not. Then there exists a pair $(m, w)$ for which

$$
u(m, w)>U\left(m ; x_{*}\right) \text { and } v(m, w)>V\left(m ; x_{*}\right) .
$$

Because $x_{k}$ converges to $x_{*}$, there exists $K$ such that $u(m, w)>U\left(m ; x_{k}\right)$ and $v(m, w)>V\left(m ; x_{k}\right)$ holds for all $k>K$. From (30), this implies

$$
x_{k}(m, w)=\lambda_{k} c\left(\sigma_{x_{k}}, \tau_{x_{k}}\right) x_{k}(m, m) x_{k}(w, w),
$$

for such $k$.

From the definition of payoffs in (5) and (6) we can write

$$
\begin{aligned}
x_{k}(m, m) U\left(m ; x_{k}\right) & =\sum_{w \in W} x_{k}(m, w)\left[u(m, w)-U\left(m ; x_{k}\right)\right], \\
x_{k}(w, w) V\left(w ; x_{k}\right) & =\sum_{m \in M} x_{k}(m, w)\left[v(m, w)-V\left(w ; x_{k}\right)\right] .
\end{aligned}
$$

Using (30) to infer

$$
\begin{aligned}
x_{k}(m, w)\left[u(m, w)-U\left(m ; x_{k}\right)\right] & =x_{k}(m, w) \max \left[0, u(m, w)-U\left(m ; x_{k}\right)\right], \\
x_{k}(m, w)\left[v(m, w)-V\left(w ; x_{k}\right)\right] & =x_{k}(m, w) \max \left[0, v(m, w)-V\left(w ; x_{k}\right)\right],
\end{aligned}
$$

yields

$$
\begin{aligned}
& x_{k}(m, m) U\left(m ; x_{k}\right)=\sum_{w^{\prime} \in W} x_{k}\left(m, w^{\prime}\right) \max \left[0, u\left(m, w^{\prime}\right)-U\left(m ; x_{k}\right)\right], \\
& x_{k}(w, w) V\left(w ; x_{k}\right)=\sum_{m^{\prime} \in M} x_{k}\left(m^{\prime}, w\right) \max \left[0, v\left(m^{\prime}, w\right)-V\left(w ; x_{k}\right)\right],
\end{aligned}
$$

\footnotetext{
${ }^{25}$ Let $\left(f_{k}, g_{k}, \alpha_{k}\right)$ be an equilibrium inducing $x_{k}$. Recall that we have set $\delta=\eta=1$. From (26) - (27), we thus have $f_{k}(m)=x_{k}(m, m)$ and $g_{k}(w)=x_{k}(w, w)$. Substituting into (12) and (13), shows that the interpretation of the following expressions as meeting rates is appropriate.

${ }^{26} \mathrm{This}$ assumption is without loss of generality: If $x_{*}$ is a limit matching with the sequence $x_{k}$ converging to it, then there exists a subsequence of $x_{k}$ such that the limits $\phi_{*}(m)$ and $\gamma_{*}(w)$ are well-defined.
} 
and, thus,

$$
\begin{gathered}
x_{k}(m, m) U\left(m ; x_{k}\right) \geq x_{k}(m, w)\left[u(m, w)-U\left(m ; x_{k}\right)\right], \\
x_{k}(w, w) V\left(w ; x_{k}\right) \geq x_{k}(m, w)\left[v(m, w)-V\left(w ; x_{k}\right)\right] .
\end{gathered}
$$

Using (B.4), $x_{k}(h, h)>0$ for all $h \in M \cup W$ (cf. the proof of Lemma 7), and the definitions of $\phi_{k}(m)$ and $\gamma_{k}(w)$ in (B.1) and (B.2), this implies

$$
\begin{aligned}
& U\left(m ; x_{k}\right) \geq \gamma_{k}(w)\left[u(m, w)-U\left(m ; x_{k}\right)\right], \\
& V\left(w ; x_{k}\right) \geq \phi_{k}(m)\left[v(m, w)-V\left(w ; x_{k}\right)\right] .
\end{aligned}
$$

Because $U\left(m ; x_{k}\right)$ and $V\left(w ; x_{k}\right)$ converge to the finite limits satisfying (B.3), these inequalities imply

$$
\phi_{*}(m)<\infty \text { and } \gamma_{*}(w)<\infty .
$$

It is immediate from (B.1), (B.2), and (B.5) that either $x_{*}(m, m)=x_{*}(w, w)=0$ must hold or that $z_{*}=\lim _{k \rightarrow \infty} \lambda_{k} c\left(\sigma_{x_{k}}, \tau_{x_{k}}\right)$ exists and is finite. The following argument excludes the second possibility. Finiteness of $z_{*}$ implies that $\phi_{*}\left(m^{\prime}\right)<\infty$ and $\gamma_{*}\left(w^{\prime}\right)<\infty$ holds for all $m^{\prime} \in M$ and $w^{\prime} \in W$. Using (29), this implies $x_{*}\left(m^{\prime}, w^{\prime}\right)=0$ whenever $x_{*}\left(m^{\prime}, m^{\prime}\right)=0$ or $x_{*}\left(w^{\prime}, w^{\prime}\right)=0$ holds (because $x_{*}\left(m^{\prime}, w^{\prime}\right) \leq \phi_{*}\left(m^{\prime}\right) x_{*}\left(w^{\prime}, w^{\prime}\right)$ and $\left.x_{*}\left(m^{\prime}, w^{\prime}\right) \leq \gamma_{*}\left(w^{\prime}\right) x_{*}\left(m^{\prime}, m^{\prime}\right)\right)$. Consequently, $x_{*}\left(m^{\prime}, m^{\prime}\right)=0$ implies $x_{*}\left(m^{\prime}, m^{\prime}\right)=1-\sum_{w^{\prime} \in W} x_{*}\left(m^{\prime}, w^{\prime}\right)=1$, which is a contradiction. Hence, finiteness of $z_{*}$ implies that $x_{*}\left(m^{\prime}, m^{\prime}\right)>0$ holds for all $m^{\prime} \in M$. Similarly, we obtain $x_{*}\left(w^{\prime}, w^{\prime}\right)>0$ for all $w^{\prime} \in W$. In particular, we have $\sigma_{x_{*}}=\sum_{m^{\prime} \in M} x_{*}(m, m)>0$ and $\tau_{x_{*}}=\sum_{w^{\prime} \in W} x_{*}\left(w^{\prime}, w^{\prime}\right)>0$. Because the contact function $C$ is strictly positive whenever both of its arguments are strictly positive, we obtain $c\left(\sigma_{x_{*}}, \tau_{x_{*}}\right)>0$. Hence, $z_{*}=\lim _{k \rightarrow \infty} \lambda_{k} c\left(\sigma_{x_{k}}, \tau_{x_{k}}\right)=\infty$, which is a contradiction.

Hence, (B.5) implies $x_{*}(m, m)=0$ and $x_{*}(w, w)=0$ and, thus (from (29)), $x_{*}(m, w)=0$. From Lemma 2, as $x_{*}$ is regret-free, $x_{*}(m, m)=0$ and $x_{*}(w, w)=0$ imply that there exist $w^{\prime}$ and $m^{\prime}$ such that $x_{*}\left(m, w^{\prime}\right)=1$ and $x_{*}\left(m^{\prime}, w\right)=1$. Using (29) again, we have $x_{k}\left(m, w^{\prime}\right) \leq$ $\phi_{k}(m) x_{k}\left(w^{\prime}, w^{\prime}\right)$ for all $k$, so that $x_{*}\left(m, w^{\prime}\right)=1$ and $x_{*}\left(w^{\prime}, w^{\prime}\right)=0$ (which is implied by $x_{*}\left(m, w^{\prime}\right)=$ 1) implies $\phi_{*}(m)=\infty$. (An analogous argument implies $\gamma_{*}(w)=\infty$.) This is a contradiction to (B.5). Hence, $x_{*}$ is pairwise stable.

Lemma 9. Let $x_{*}$ be a coherent and regret-free matching. Then there exists $\lambda_{e}>0$ such that for all $\lambda>\underline{\lambda}_{e}$, an equilibrium matching $x^{\lambda}$ exists and $\lim _{\lambda \rightarrow \infty} x^{\lambda}=x_{*}$ holds. In particular, $x_{*}$ is a limit matching.

Proof. Let $x_{*}$ be a coherent and regret-free matching. For any $r \in \mathbb{R}_{+}$define the matching $x_{r}$ given by

$$
x_{r}(m, w)= \begin{cases}x_{*}(m, w) & \text { if } x_{*}(m, w)<1, \\ \left(1-\frac{1}{r+1}\right) & \text { if } x_{*}(m, w)=1 .\end{cases}
$$

Because $x_{*}$ is a matching, $x_{r}$ satisfies (1) - (3). Thus, $x_{r}$ is a matching. Furthermore, we have

$$
\lim _{r \rightarrow \infty} x_{r}=x_{*} .
$$

In the following we will show that for any sufficiently large $\lambda \in \mathbb{R}_{+}$we can find a value $r(\lambda) \in \mathbb{R}_{+}$ such that

$$
x^{\lambda}:=x_{r(\lambda)}
$$


is an equilibrium matching for the search model with parameters $(1,1, \lambda, C)$. Furthermore, we will show that

$$
\lim _{\lambda \rightarrow \infty} r(\lambda)=\infty
$$

holds. Combining (B.7) and (B.8) we have $\lim _{\lambda \rightarrow \infty} x^{\lambda}=x_{*}$, proving the lemma.

We define the function $h: \mathbb{R}_{+} \times \mathbb{R}_{+} \rightarrow \mathbb{R}$ by

$$
h(\lambda, r)=\lambda\left(\frac{1}{r+1}\right)^{2} c\left(\sigma_{x_{r}}, \tau_{x_{r}}\right)-\left(1-\frac{1}{r+1}\right) .
$$

As $x_{*}$ is regret-free, Lemma 2 ensures that $\sigma_{x_{r}}>0$ and $\tau_{x_{r}}>0$ holds for all $r{ }^{27}$ ensuring that $c\left(\bar{x}_{r}^{M}, \bar{x}_{r}^{W}\right)$ is well-defined and strictly positive for all $r$. In addition, as the mapping $r \rightarrow x_{r}$ is continuous and $c$ is continuous, it follows that $h$ is a continuous function. Next, we argue that for any $\lambda \in \mathbb{R}_{++}$we have

$$
h(\lambda, 0)>0>-1=\lim _{r \rightarrow \infty} h(\lambda, r) .
$$

The first inequality in (B.10) is immediate from $c\left(\sigma_{x_{0}}, \tau_{x_{0}}\right)>0$. It remains to establish the equality in (B.10) which is equivalent to

$$
\lim _{r \rightarrow \infty}\left(\frac{1}{r+1}\right)^{2} c\left(\sigma_{x_{r}}, \tau_{x_{r}}\right)=0
$$

Suppose $\sigma_{x_{*}} \cdot \tau_{x_{*}}>0$ holds, ensuring that $c\left(\sigma_{x_{*}}, \tau_{x_{*}}\right)$ is well-defined. From (B.7) and the continuity of $c$, this implies (B.11). Suppose $\sigma_{x_{*}}=0$. As $x_{*}$ is regret-free, Lemma 2 then implies that $x_{*}$ is a simple matching. From (B.6), it then follows that $x_{r}(m, m)=1 /(r+1)$ holds for all $m \in M$ and $x_{r}(w, w) \in\{1 /(r+1), 1\}$ holds for all $w \in W$. Hence, we have $\sigma_{x_{r}}=|M| /(r+1)$ and $\tau_{x_{r}} \geq|W| /(r+1)$, implying

$$
\left(\frac{1}{r+1}\right)^{2} c\left(\sigma_{x_{r}}, \tau_{x_{r}}\right)=\left(\frac{1}{r+1}\right)^{2} \frac{C\left(\sigma_{x_{r}}, \tau_{x_{r}}\right)}{\sigma_{x_{r}} \cdot \tau_{x_{r}}} \leq \frac{1}{|M||W|} C\left(\sigma_{x_{r}}, \tau_{x_{r}}\right)
$$

where we have used the definition of $c$ in (11) for the equality. As the contact function $C$ is continuous, satisfies $C\left(0, \tau_{x_{*}}\right)=0$, and $x_{r}$ converges to $x_{*}$, this implies (B.11). An analogous argument establishes (B.11) for the case $\tau_{x_{*}}=0$. Hence, (B.10) holds.

From (B.10) and the continuity of $h$ in $r$ it follows that for every $\lambda>0$ there exists $r(\lambda)$ such that

$$
h(\lambda, r(\lambda))=0
$$

holds. As $\lim _{\lambda \rightarrow \infty} h(\lambda, r)=\infty$ holds for any given $r \in \mathbb{R}$ we have (B.8). It remains to establish that for sufficiently large $\lambda$ the matching $x^{\lambda}$ is an equilibrium matching. We begin by establishing that for sufficiently large $\lambda$, the matching $x^{\lambda}$ is a steady-state matching.

Let $\underline{\lambda}_{s}$ be such that $x_{*}(h, h)>1 /\left(r(\lambda+1)\right.$ holds for all $\lambda>\underline{\lambda}_{s}$ and $h \in M \cup W$ for which $x_{*}(h, h)>0$ holds. (As there is a finite number of types and (B.8) holds, the existence of such a

\footnotetext{
${ }^{27}$ If there exists $(m, w)$ such that $x_{*}(m, w)=1$ holds, then we have $x_{r}(m, w)=1-1 /(r+1), x_{k}\left(m, w^{\prime}\right)=0$, and $x_{k}\left(m^{\prime}, w\right)=0$ for all $w^{\prime} \neq w$ and $m^{\prime} \neq m$. Consequently, $x_{r}(m, m)=x_{r}(w, w)=1 /(r+1)>0$ holds, implying $\sigma_{x_{r}}>0$ and $\tau_{x_{r}}>0$. If there exists no $(m, w)$ such that $x_{*}(m, w)=1$ holds, then (B.6) implies $x_{r}=x_{*}$. In addition, Lemma 2 implies that no agent is fully matched in $x_{*}$. Consequently, $\sigma_{x_{*}}=\sigma_{x_{r}}>0$ and $\tau_{x_{*}}=\tau_{x_{r}}>0$ hold.
} 
$\underline{\lambda}_{s}$ is assured.) We now show that for all $\lambda>\underline{\lambda}_{s}$ the matching $x^{\lambda}$ is a steady-state matching for the search model with parameters $(1,1, \lambda, C)$. Using Lemma 7 , we have to show

$$
x^{\lambda}(m, w) \leq \lambda c\left(\sigma_{x^{\lambda}}, \tau_{x^{\lambda}}\right) x^{\lambda}(m, m) x^{\lambda}(w, w)
$$

for all $(m, w) \in M \times W$. Consider $(m, w)$ satisfying $x_{*}(m, w)=1$. Then

$$
\frac{x^{\lambda}(m, w)}{\lambda \cdot c\left(\sigma_{x^{\lambda}}, \tau_{x^{\lambda}}\right) x^{\lambda}(m, m) x^{\lambda}(w, w)}=\frac{1-\frac{1}{r(\lambda)+1}}{\lambda \cdot c\left(\sigma_{x^{\lambda}}, \tau_{x^{\lambda}}\right)\left(\frac{1}{r(\lambda)+1}\right)^{2}}=1,
$$

where the first equality uses (B.6) to infer $x^{\lambda}(m, w)=1-1 /(r(\lambda)+1)$ as well as $x^{\lambda}(m, m)=$ $x^{\lambda}(w, w)=1 /(r(\lambda)+1)$ from $x_{*}(m, w)=1$, and the second equality is from (B.12). It follows that (B.13) holds with equality. Consider $(m, w)$ satisfying $0<x_{*}(m, w)<1$. As $x_{*}$ is regret-free, Lemma 2 and (B.6) then imply $x_{*}(m, m)=x^{\lambda}(m, m)>0$ and $x_{*}(w, w)=x^{\lambda}(w, w)>0$. Hence,

$$
\begin{aligned}
\frac{x^{\lambda}(m, w)}{\lambda \cdot c\left(\sigma_{x^{\lambda}}, \tau_{x^{\prime}}\right) x^{\lambda}(m, m) x^{\lambda}(w, w)} & =\frac{x_{*}(m, w)}{\lambda \cdot c\left(\sigma_{x^{\lambda}}, \tau_{x^{\prime}}\right) x_{*}(m, m) x_{*}(w, w)} \\
& <\frac{1-\frac{1}{r(\lambda)+1}}{\lambda \cdot c\left(\sigma_{x^{\lambda}}, \tau_{x^{\prime}}\right)\left(\frac{1}{r(\lambda)+1}\right)^{2}} \\
& =1,
\end{aligned}
$$

where the inequality uses that $\lambda>\underline{\lambda}_{s}$ implies $x_{*}(m, m)>1 /(r(\lambda)+1), x_{*}(w, w)>1 /(r(\lambda)+1)$, and $x^{*}(m, w)<1-1 /(r(\lambda)+1)$. It follows that (B.13) holds with strict inequality. Finally, from (B.6), $x_{*}(m, w)=0$ implies $x^{\lambda}(m, w)=0$, so that (B.13) trivially holds for all $(m, w)$ satisfying $x_{*}(m, w)=0$.

Using Lemma 7, it remains to show that there exists $\underline{\lambda}_{e} \geq \underline{\lambda}_{s}$ such that for $\lambda>\underline{\lambda}_{e}$ the condition

$$
x^{\lambda}(m, w)= \begin{cases}0 & \text { if } u(m, w)<U\left(m ; x^{\lambda}\right) \text { or } v(m, w)<V\left(w ; x^{\lambda}\right), \\ \lambda c\left(\sigma_{x^{\lambda}}, \tau_{x^{\lambda}}\right) x^{\lambda}(m, m) x^{\lambda}(w, w) & \text { if } u(m, w)>U\left(m ; x^{\lambda}\right) \text { and } v(m, w)>V\left(w ; x^{\lambda}\right)\end{cases}
$$

hold for all $(m, w) \in M \times W$.

From (B.6) the payoffs associated with $x^{\lambda}$ are given by

$$
U\left(m ; x^{\lambda}\right)= \begin{cases}\left(1-\frac{1}{r(\lambda)+1}\right) U\left(m ; x_{*}\right) & \text { if } x_{*}(m, m)=0, \\ U\left(m ; x_{*}\right) & \text { if } x_{*}(m, m)>0,\end{cases}
$$

and by

$$
V\left(w ; x^{\lambda}\right)= \begin{cases}\left(1-\frac{1}{r(\lambda)+1}\right) V\left(w ; x_{*}\right) & \text { if } x_{*}(w, w)=0 \\ V\left(w ; x_{*}\right) & \text { if } x_{*}(w, w)>0 .\end{cases}
$$

In both cases, Lemma 2 is used to exclude the possibility that $x^{*}(h, h)=0$ holds when $h$ has more than one partner in $x^{*}$.

From (B.7), the continuity of the payoff functions (or directly from (B.16) - (B.17)), and (B.8), there exists $\underline{\lambda}_{e} \geq \underline{\lambda}_{s}$ such that for all $\lambda>\lambda_{e}$ the following implications hold for all $m \in M$ and $w \in W$ :

$$
\begin{gathered}
u(m, w)<U\left(m ; x_{*}\right) \Rightarrow u(m, w)<U\left(m ; x^{\lambda}\right), \\
v(m, w)<V\left(w ; x_{*}\right) \Rightarrow v(m, w)<V\left(w ; x^{\lambda}\right),
\end{gathered}
$$


Fix any $\lambda>\underline{\lambda}_{e}$. To show that (B.15) holds for all $(m, w) \in M \times W$, we consider three cases and establish that (B.15) holds in each of these cases. As each pair ( $m, w) \in M \times W$ belongs to at least one of these cases, this finishes the proof.

CASe 1: $x_{*}(m, m)=1$ or $x_{*}(w, w)=1$ : Consider $m$ satisfying $x_{*}(m, m)=1$. We then have $x_{*}(m, w)=x^{\lambda}(m, w)=0$ for all $w \in W$, implying $U\left(m ; x_{*}\right)=U\left(m ; x^{\lambda}\right)=0$. Now consider any $w \in W$. If $u(m, w)<0$, it is immediate that (B.15) holds. Hence, suppose $u(m, w)>0$. We have to show that $v(m, w) \leq V\left(w ; x^{\lambda}\right)$ holds, as this implies that $x^{\lambda}(m, w)=0$ is consistent with (B.15). If $x_{*}(w, w)>0$, then the inequality $v(m, w) \leq V\left(w ; x^{\lambda}\right)$ is immediate from $V\left(w ; x^{\lambda}\right)=$ $V\left(w ; x_{*}\right)$ and the pairwise stability of $x_{*}$. If $x_{*}(w, w)=0$, the pairwise stability of $x_{*}$ implies $v(m, w)<V\left(w ; x_{*}\right)$. (To obtain the strict inequality here, we use the strictness of preferences together with the implication from Lemma 2 that there exists $m^{\prime} \neq m$ such that $x_{*}\left(m^{\prime}, w\right)=1$, implying $V\left(w ; x_{*}\right)=v\left(m^{\prime}, w\right)$ for some $m^{\prime} \neq m$.) The desired result then follows from (B.19). 1 .

An analogous argument using (B.18) shows that (B.15) holds for all $(m, w)$ satisfying $x_{*}(w, w)=$

CASe 2: $x_{*}(m, m)=0$ oR $x_{*}(w, w)=0$ : Consider $m$ satisfying $x_{*}(m, m)=0$. Lemma 2 then implies that there exists $w^{\prime} \in W$ such that $x_{*}\left(m, w^{\prime}\right)=1$ and, thus, $U\left(m ; x_{*}\right)=u\left(m, w^{\prime}\right)$ as well as $V\left(w^{\prime} ; x_{*}\right)=v\left(m, w^{\prime}\right)$. By the individual rationality of $x_{*}$ and the strictness of preferences, we have $u\left(m, w^{\prime}\right)>0$ and $v\left(m, w^{\prime}\right)>0$. From (B.16) and (B.17) we then have $u\left(m, w^{\prime}\right)>U\left(m ; x^{\lambda}\right)$ and $v\left(m, w^{\prime}\right)>V\left(w^{\prime} ; x^{\lambda}\right)$. Consequently, (B.15) requires that (B.13) holds with equality for the pair $\left(m, w^{\prime}\right)$, which is ensured by construction of $r(\lambda)$, cf. (B.14). Consider now $w \neq w^{\prime}$. Because $x_{*}\left(m, w^{\prime}\right)=1$ we have $x_{*}(m, w)=0$ and, thus, $x^{\lambda}(m, w)=0$. We have to show that $x^{\lambda}(m, w)=0$ is consistent with (B.15). If $u(m, w)<U\left(m ; x_{*}\right)$, then (B.18) implies $u(m, w)<$ $U\left(m ; x^{\lambda}\right)$, establishing the desired conclusion. If $u(m, w)>U\left(m ; x_{*}\right)$ (the case of equality cannot arise because $U\left(m ; x_{*}\right)=u\left(m, w^{\prime}\right)$ holds for some $w \neq w^{\prime}$ and preferences are strict), the pairwise stability of $x_{*}$ implies $v(m, w)<V\left(w ; x_{*}\right)$. By (B.19) this implies $v(m, w)<V\left(w ; x^{\lambda}\right)$, establishing the desired conclusion. Hence, we conclude that (B.15) holds for all $(m, w)$ with $x_{*}(m, m)=0$.

An analogous argument shows that (B.15) holds for all $(m, w)$ satisfying $x_{*}(w, w)=0$.

CASE 3: $0<x_{*}(m, m)<1$ AND $0<x_{*}(w, w)<1$ : Using (B.6), (B.16), and (B.17), we have $x^{\lambda}(m, w)=x_{*}(m, w), U\left(m ; x^{\lambda}\right)=U\left(m, x_{*}\right)$, and $V\left(w ; x^{\lambda}\right)=V\left(w ; x_{*}\right)$. Pairwise stability of $x_{*}$ then implies that there does not exist such $(m, w)$ satisfying $u(m, w)>U\left(m ; x^{\lambda}\right)$ and $v(m, w)>$ $V\left(m ; x^{\lambda}\right)$. Because $x_{*}$ is regret-free, $x^{\lambda}(m, w)>0$ also implies $u(m, w) \geq U\left(m ; x^{\lambda}\right)$ as well as $v(m, w) \geq V\left(w ; x^{\lambda}\right)$. Therefore, (B.15) holds for all $(m, w)$ satisfying $0<x_{*}(m, m)<1$ and $0<x_{*}(w, w)<1$.

\section{Appendix C. Proof of Proposition 2}

In the following, it is often more convenient to identify simple matchings with their matchings functions, instead of using the assignment matrices (cf. Section 2.2).

We establish Proposition 2 through a sequence of lemmas. We begin by establishing that the existence of a coherent and regret-free fractional matching implies the existence of multiple stable simple matchings. By Theorem 1, this proves one direction of the equivalence claimed in Proposition 2.

Lemma 10. If there exists a fractional matching $x$ that is coherent and regret-free, then there exist two distinct stable simple matchings $\mu_{1} \neq \mu_{2}$. 
Proof. Let $x$ be a fractional matching that is coherent and regret-free. Let

$$
P_{x}(m)=\{w \in W: x(m, w)>0\} \text { and } P_{x}(w)=\{m \in M: x(m, w)>0\}
$$

denote the sets of partners in this matching. We now construct $\mu_{1}$ and $\mu_{2}$. Let $\mu_{1}(h)=h$ if $x(h, h)=1$. For all other agents, let $\mu_{1}(m)=\operatorname{argmax}_{w \in P_{x}(m)} u(m, w)$ and $\mu_{1}(w)=\operatorname{argmin}_{m \in P_{x}(w)} v(m, w)$, respectively. By the strictness of preferences, the function $\mu_{1}: M \cup W \rightarrow M \cup W$ is uniquely defined. Similarly, let $\mu_{2}(h)=h$ if $x(h, h)=1$. For all other agents, let $\mu_{2}(m)=\operatorname{argmin}_{w \in P_{x}(m)} u(m, w)$ and $\mu_{2}(w)=\operatorname{argmax}_{m \in P_{x}(w)} v(m, w)$, respectively. We show in the following that $\mu_{1}$ and $\mu_{2}$ are stable simple matchings that satisfy $\mu_{1} \neq \mu_{2}$.

We begin by verifying that $\mu_{1}$ and $\mu_{2}$ are simple matchings. It is immediate from the definitions that for $i=1,2$ the conditions $\mu_{i}(m) \in W \cup\{m\}$ and $\mu_{i}(w) \in M \cup\{w\}$ are satisfied for all $m$ and $w$. Hence, our task is to verify that for $i=1,2$ the condition $\mu_{i}^{2}(h)=h$ holds for all $h \in M \cup W$. For $h$ satisfying $x(h, h)=1$ this is immediate. Consider $h$ satisfying $x(h, h)=0$. Because $x$ is regret-free, Lemma 2 implies that every such agent has a unique partner $h^{\prime} \neq h$ and $h^{\prime}$ is in turn the unique partner of $h$. Consequently, we have $\mu_{1}^{2}(h)=\mu_{2}^{2}(h)=h$ for all such agents. It remains to consider $h$ satisfying $0<x(h, h)<1$, that is, the set of partially matched agents.

Let $M_{x}$ and $W_{x}$ denote the sets of partially matched men and women, respectively, in the matching $x$. As $x$ is regret-free, Lemma 2 implies that these sets are not empty. Because every agent in $M_{x} \cup W_{x}$ has at least one partner (otherwise the agent would be unmatched), the set of partners $P_{x}(h)$ is not empty for all $h \in M_{x} \cup W_{x}$. Furthermore, for all $h \in M_{x} \cup W_{x}$ we have $P_{x}(h) \subset M_{x} \cup W_{x}$. (If, say, $m \in M_{x}$ has a partner $w^{\prime} \in W \backslash W_{x}$, then $w^{\prime}$ must be fully matched. From Lemma 2, this implies $x\left(m, w^{\prime}\right)=1$, implying $m \notin M_{x}$.) Hence, for $i=1,2$ we have $\mu_{i}\left(M_{x}\right) \subseteq W_{x}$ and $\mu_{i}\left(W_{x}\right) \subseteq M_{x}$.

We use the individual rationality of $x$ for the first inequalities in the following displayed expressions,

$$
\begin{gathered}
u\left(m, \mu_{1}(m)\right)>(1-x(m, m)) u\left(m, \mu_{1}(m)\right) \geq \sum_{w \in P_{x}(m)} x(m, w) u(m, w)=U(m ; x), \\
v\left(\mu_{2}(w), w\right)>\left(1-x(w, w) v\left(\mu_{2}(w), w\right) \geq \sum_{m \in P_{x}(w)} x(m, w) v(m, w)=V(w ; x),\right.
\end{gathered}
$$

for all $m \in M_{x}$ and $w \in W_{x}$. Hence, we have

$$
u\left(m, \mu_{1}(m)\right)>U(m ; x) \text { and } v\left(\mu_{2}(w), w\right)>V(w ; x),
$$

for all $m \in M_{x}$ and $w \in W_{x}$.

Because $x$ is pairwise stable, (C.1) implies

$$
v\left(m, \mu_{1}(m)\right) \leq V\left(\mu_{1}(m) ; x\right) \text { and } u\left(\mu_{2}(w), w\right) \leq U\left(\mu_{2}(w) ; x\right)
$$

for all $m \in M_{x}$, respectively for all $w \in W_{x}$.

Because $x$ is regret free, these inequalities must, in fact, hold with equality, so that we obtain

$$
v\left(m, \mu_{1}(m)\right)=V\left(\mu_{1}(m) ; x\right) \text { and } u\left(\mu_{2}(w), w\right)=U\left(\mu_{2}(w) ; x\right),
$$

for all $m \in M_{x}$ and $w \in W_{x}$. From the first equality in (C.2), $\mu_{1}(m)=\mu_{1}\left(m^{\prime}\right)=w$ implies $v\left(m^{\prime}, w\right)=v(m, w)$ and thus, from strictness of preferences, $m=m^{\prime}$. Hence, the restriction of $\mu_{1}$ to $M_{x}$, denoted by $\eta_{1}$ in the following, is an injection into $W_{x}$. An analogous argument 
using the second equality in (C.2) shows that the restriction of $\mu_{2}$ to $W_{x}$, denoted by $\eta_{2}$, is an injection into $M_{x}$. Because the sets $M_{x}$ and $W_{x}$ are finite it follows (as a trivial application of the Cantor-Bernstein-Schröder theorem) that $\eta_{1}$ and $\eta_{2}$ are bijections. Let $\eta_{1}^{-1}: W_{x} \rightarrow M_{x}$ and $\eta_{2}^{-1}: M_{x} \rightarrow W_{x}$ denote the corresponding inverses. To establish that $\mu_{1}$ and $\mu_{2}$ are simple matchings, it remains to show that $\eta_{1}^{-1}(w)=\mu_{1}(w)$ and $\eta_{2}^{-1}(m)=\mu_{2}(m)$ holds for all $w \in W_{x}$ and $m \in M_{x}$. Consider $w \in W_{x}$. From the first equality in (C.2), we have $v\left(\eta_{1}^{-1}(w), w\right)=V(w ; x)$. Observing that $\eta_{1}^{-1}(w)$ is a partner of $w$ and that (because $x$ is regret-free and preferences are strict) all other partners $m$ of $w$ satisfy $v(m, w)>V(w ; x)$, the desired conclusion $\eta_{1}^{-1}(w)=\mu_{1}(w)$ follows. An analogous argument yields $\eta_{2}^{-1}(m)=\mu_{2}(m)$ for all $m \in M_{x}$.

Consider any $m \in M_{x}$. Substituting $w=\mu_{1}(m)$ into the second inequality in (C.1), we have $v\left(\mu_{2}\left(\mu_{1}(m)\right), \mu_{1}(m)\right)>V\left(\mu_{1}(m) ; x\right)$. From the first equality in (C.2), this implies $\mu_{2}\left(\mu_{1}(m)\right) \neq m$. Because $M_{x}$ is not empty, it follows that $\mu_{1}$ and $\mu_{2}$ are different. To complete the proof of the lemma, it remains to show that $\mu_{1}$ and $\mu_{2}$ are stable. Let $x_{i}$ denote the assignment matrix corresponding to $\mu_{i}$. By construction, $x_{i}(m, w)=1$ implies that $(m, w)$ are partners in $x$, that is, we have $x(m, w)>0$. Because $x$ is individually rational, it follows that $\mu_{i}$ is individually rational. Next, by the construction of $\mu_{1}$ and $\mu_{2}$ we have

$$
\begin{gathered}
U\left(m ; x_{1}\right)=U\left(m ; x_{2}\right)=U(m ; x) \text { for all } m \notin M_{x}, \\
V\left(w ; x_{1}\right)=V\left(w ; x_{2}\right)=V(w ; x) \text { for all } w \notin W_{x} .
\end{gathered}
$$

From (C.1) and (C.2) we have:

$$
\begin{gathered}
U\left(m ; x_{1}\right)>U\left(m ; x_{2}\right)=U(m ; x) \text { for all } m \in M_{x}, \\
V\left(w ; x_{2}\right)>V\left(w ; x_{1}\right)=V(w ; x) \text { for all } w \in W_{x} .
\end{gathered}
$$

In particular, for $i=1,2$ we have $U\left(m ; x_{i}\right) \geq U(m ; x)$ for all $m \in M$ and $V\left(w ; x_{i}\right) \geq V(w ; x)$ for all $w \in W$. As $x$ satisfies (8) it follows that $x_{1}$ and $x_{2}$ satisfy (8). Hence, $\mu_{1}$ and $\mu_{2}$ are stable.

To prove the converse of Lemma 10 and, thus by Theorem 1, to finish the proof of Proposition 2, we rely on some well-known results about the structure of the set of stable simple matchings. Given any two simple matchings $\mu_{1}$ and $\mu_{2}$, define $\mu_{1}>_{M} \mu_{2}$ if $u\left(m, \mu_{1}(m)\right) \geq u\left(m, \mu_{2}(m)\right)$ holds for all $m \in M$ and $u\left(m, \mu_{1}(m)\right)>u\left(m, \mu_{2}(m)\right)$ for at least one $m$. Define $\mu_{1}>_{W} \mu_{2}$ in an analogous way. If $\mu_{1}$ and $\mu_{2}$ are both stable, then [26, Theorem 2.13]

$$
\mu_{1}>_{M} \mu_{2} \Leftrightarrow \mu_{2}>_{W} \mu_{1} \text {. }
$$

Two stable simple matchings $\mu_{1}$ and $\mu_{2}$ are consecutive [26, p. 61] if $\mu_{1}>_{M} \mu_{2}$ holds and there does not exist a stable simple matching $\mu_{3}$ between $\mu_{1}$ and $\mu_{2}$, that is, satisfying $\mu_{1}>_{M} \mu_{3}>_{M} \mu_{2}$. Consecutive stable simple matchings exist if and only if there is more than one stable simple matching: If there is more than one stable simple matching, the men-optimal stable matching $\mu_{M}$ and the women-optimal stable matching $\mu_{W}$ (Roth and Sotomayor [26, Definition 2.11]) satisfy $\mu_{M}>_{M} \mu_{W}$. The set of simple matchings is finite, therefore there exists a matching $\mu^{\prime}$ such that $\mu_{M}$ and $\mu^{\prime}$ are consecutive. To finish the proof of Proposition 2, it thus suffices to show that coherent and regret-free fractional matchings exist if consecutive stable simple matchings exist. The following lemmas establish this.

Lemma 11. Let $\mu_{1}$ and $\mu_{2}$ be consecutive stable simple matchings. Then there does not exist $(m, w) \in M \times W$ satisfying

$$
u\left(m, \mu_{1}(m)\right)>u(m, w)>u\left(m, \mu_{2}(m)\right) \text { and } v\left(\mu_{2}(w), w\right)>v(m, w)>v\left(\mu_{1}(w), w\right) .
$$


Proof. ${ }^{28}$ Let $\mu_{1}$ and $\mu_{2}$ be consecutive stable simple matchings for the marriage market $(M, W ; u, v)$. Consider changing all man's preferences such that $\mu_{2}(m)$ becomes the least acceptable partner in the marriage market, that is consider a marriage market $\left(M, W ; u^{\prime}, v\right)$ satisfying $u^{\prime}(m, h)=u(m, h)$ if $u(m, h) \geq u\left(m, \mu_{2}(m)\right)$ or $h=m$ holds and $u^{\prime}(m, h)<0$ otherwise for all $m \in M$. Then $\mu_{2}$ is the women-optimal matching in $\left(M, W ; u^{\prime}, v\right)$. Now take any man $m$ such that $\mu_{1}(m) \neq \mu_{2}(m)$ holds. This implies that man $m$ is matched in both $\mu_{1}$ and $\mu_{2}$. Let $\left(M ; W, u^{\prime \prime}, v\right)$ be the marriage market which differs from $\left(M, W ; u^{\prime}, v\right)$ only in that woman $\mu_{2}(m)$ is unacceptable for man $m$, that is $u^{\prime \prime}\left(m, \mu_{2}(m)\right)<0$ holds. Now consider an application of the women-proposing GaleShapley algorithm to the marriage market $\left(M, W ; u^{\prime \prime}, v\right)$, taking the matching $\mu_{2}$ as the starting point of the algorithm and beginning with a rejection step in which man $m$ rejects woman $\mu_{2}(m)$. Because the matching $\mu_{1}$ is consecutive to $\mu_{2}$, the algorithm - which corresponds to the breakmarriage algorithm from McVitie and Wilson [22] - stops at the matching $\mu_{1}$ (see the proof of Theorem 4 in McVitie and Wilson [22, Section 4.1]). Further, because $\mu_{1}(m) \neq m$, the algorithm stops the first time that man $m$ receives a proposal from a woman $w$ such that $u^{\prime \prime}(m, w)>0 \Leftrightarrow u(m, w)>u\left(m, \mu_{2}(w)\right)$ holds. It follows that man $m$ does not receive a proposal from any woman $w$ satisfying the first condition in (C.4). On the other hand, every woman $w$ for which the second condition in (C.4) holds, must make a proposal to $m$. It follows that there does not exists $(m, w) \in M \times W$ satisfying (C.4).

We say that the simple matchings $\mu_{1}$ and $\mu_{2}$ support the fractional matching $x$ if the assignment matrices $x_{1}$ and $x_{2}$ associated with $\mu_{1}$ and $\mu_{2}$ satisfy

$$
\begin{aligned}
& x(m, w)=0 \Leftrightarrow\left[x_{1}(m, w)=0 \text { and } x_{2}(m, w)=0\right], \\
& x(m, w)=1 \Leftrightarrow\left[x_{1}(m, w)=1 \text { and } x_{2}(m, w)=1\right],
\end{aligned}
$$

for all $(m, w) \in M \times W$

Lemma 12. Suppose the fractional matching $x$ is supported by the consecutive stable simple matchings $\mu_{1}$ and $\mu_{2}$. If

$$
U(m ; x)=u\left(m, \mu_{2}(m)\right) \text { and } V(w ; x)=v\left(\mu_{1}(w), w\right)
$$

holds for all $m \in M$ and $w \in W$, then $x$ is coherent and regret-free.

Proof. Let $x, \mu_{1}$ and $\mu_{2}$ satisfy the conditions in the statement of the lemma, and let $x_{1} \neq x_{2}$ denote the assignment matrices associated with $\mu_{1}$ and $\mu_{2}$.

Because $\mu_{1}$ and $\mu_{2}$ support $x$, (C.5) implies that $x(m, w)>0$ only holds if either $x_{1}(m, w)=1$ or $x_{2}(m, w)=1$ (or both) hold. As $x_{1}$ and $x_{2}$ are individually rational this implies $u(m, w)>0$. Hence, $x$ is individually rational.

From (C.6) for any pair $(m, w)$ that satisfies $x(m, w)=1$, it must be that $U(m ; x)=u(m, w)$ and $V(w ; x)=v(m, w)$. Hence, condition (9) holds for such pairs. To verify that $x$ is regretfree, it remains to consider pairs $(m, w)$ for which $0<x(m, w)<1$. From (C.5) and (C.6), either $x_{1}(m, w)=1$ or $x_{2}(m, w)=1$ (but not both) hold. Consider the first of these cases. In this case, $u(m, w)=u\left(m, \mu_{1}(m)\right) \geq u\left(m, \mu_{2}(m)\right)$ and $v(m, w)=v\left(\mu_{1}(w), w\right)$, where the inequality is from $\mu_{1}>_{M} \mu_{2}$. Condition (C.7) then implies $u(m, w) \geq U(m ; x)$ and $v(m, w)=V(w ; x)$,

\footnotetext{
${ }^{28} \mathrm{We}$ are grateful for an anonymous referee who suggested the following proof based on the logic of the breakmarriage algorithm from McVitie and Wilson [22]
} 
establishing that (9) holds. In the second case, it must be that $u(m, w)=u\left(m, \mu_{2}(m)\right)$ and $v(m, w)=v\left(\mu_{2}(w), w\right) \geq v\left(\mu_{1}(w), w\right)$, where the inequality is from $\mu_{1}>_{M} \mu_{2}$ and (C.3). As in the previous case, (C.7) then implies (9). Hence, $x$ is regret-free.

It remains to show the pairwise stability of $x$. Suppose, to the contrary, that there is a pair $(m, w) \in M \times W$ violating (8). Condition (C.7) then implies

$$
u(m, w)>u\left(m, \mu_{2}(m)\right) \text { and } v(m, w)>v\left(\mu_{1}(w), w\right) .
$$

By the stability of $\mu_{2}$, the first of these inequalities implies $v\left(\mu_{2}(w), w\right) \geq v(m, w)$, whereas from stability of $\mu_{1}$ the second inequality implies $u\left(m, \mu_{1}(m)\right) \geq u(m, w)$. From Lemma 11, at the most one of the inequalities $v\left(\mu_{2}(w), w\right) \geq v(m, w)$ and $u\left(m, \mu_{1}(m)\right) \geq u(m, w)$ can be strict. Suppose the first inequality holds with equality. Because of the strictness of preferences, this requires $\mu_{2}(w)=m$, and, therefore, $U\left(m, \mu_{2}(m)\right)=u(m, w)$, which contradicts the first inequality in (C.8). Similarly, if $u\left(m, \mu_{1}(m)\right)=u(m, w)$ holds, then $v(m, w)=v\left(\mu_{1}(w), w\right)$, which contradicts the second inequality in (C.8). Therefore, there is no pair $(m, w)$ satisfying (C.8), proving that $x$ is pairwise stable.

To conclude the proof of Proposition 2, it remains to show that if $\mu_{1}$ and $\mu_{2}$ are consecutive stable simple matchings, then there exists a fractional matching $x$ that is supported by $\mu_{1}$ and $\mu_{2}$ and satisfies (C.7). This is implied by the following lemma, which proves the stronger result that such a fractional matching $x$ exists whenever $\mu_{1}>_{M} \mu_{2}$ holds.

Lemma 13. Let $\mu_{1}$ and $\mu_{2}$ be stable simple matchings such that $\mu_{1}>_{M} \mu_{2}$. Then there exists a fractional matching $x$ that is supported by $\mu_{1}$ and $\mu_{2}$ and satisfies conditions (C.7).

Proof. Let $\tilde{M}=\left\{m \in M \mid \mu_{1}(m) \neq \mu_{2}(m)\right\}$ and $\tilde{W}=\left\{w \in W \mid \mu_{1}(w) \neq \mu_{2}(w)\right\}$. As $\mu_{1} \neq \mu_{2}$, we have $\tilde{M} \neq \emptyset$ and $\tilde{W} \neq \emptyset$. As $\mu_{1}$ and $\mu_{2}$ are both stable, the set of unmatched agents in these matchings is the same (Roth and Sotomayor [26, Theorem 2.22]). Therefore, $\mu_{i}(\tilde{M})=\tilde{W}$ and $\mu_{i}(\tilde{W})=\tilde{M}$ hold for $i=1,2$. Define an oriented graph whose nodes are $\tilde{M} \cup \tilde{W}$ as follows: (i) there is an arc from $m \in \tilde{M}$ to $w \in \tilde{W}$ if $\mu_{1}(m)=w$, and (ii) there is an arc from $w \in \tilde{W}$ to $m \in \tilde{M}$ if $\mu_{2}(w)=m$. Because every node in this finite graph has a unique direct successor and a unique direct predecessor, it follows that the graph is the union of a set of disjoint directed cycles. Let $m_{1} w_{1} m_{2} w_{2} \ldots m_{\ell} w_{\ell} m_{\ell+1}$ with $m_{\ell+1}=m_{1}$ be such a cycle and consider the set of equations

$$
\begin{aligned}
u\left(m_{1}, w_{\ell}\right) & =x\left(m_{1}, w_{\ell}\right) u\left(m_{1}, w_{\ell}\right)+x\left(m_{1}, w_{1}\right) u\left(m_{1}, w_{1}\right) \\
v\left(m_{1}, w_{1}\right) & =x\left(m_{1}, w_{1}\right) v\left(m_{1}, w_{1}\right)+x\left(m_{2}, w_{1}\right) v\left(m_{2}, w_{1}\right) \\
u\left(m_{2}, w_{1}\right) & =x\left(m_{2}, w_{1}\right) u\left(m_{2}, w_{1}\right)+x\left(m_{2}, w_{2}\right) u\left(m_{2}, w_{2}\right) \\
v\left(m_{2}, w_{2}\right) & =x\left(m_{2}, w_{2}\right) v\left(m_{2}, w_{2}\right)+x\left(m_{3}, w_{2}\right) v\left(m_{3}, w_{2}\right) \\
\ldots \ldots & \\
v\left(m_{\ell}, w_{\ell}\right) & =x\left(m_{\ell}, w_{\ell}\right) v\left(m_{\ell}, w_{\ell}\right)+x\left(m_{\ell+1}, w_{\ell}\right) v\left(m_{\ell+1}, w_{\ell}\right) .
\end{aligned}
$$

Because the direct predecessor of each node is distinct from its direct successor, it must be that $\ell>1$. For $i=1, \cdots, \ell$ the following inequalities are satisfied:

$$
\begin{gathered}
u\left(m_{i}, w_{i}\right)>u\left(m_{i}, w_{i-1}\right)>0, \\
v\left(m_{i+1}, w_{i}\right)>v\left(m_{i}, w_{i}\right)>0,
\end{gathered}
$$

where we set $w_{0}=w_{\ell}$, and where the first inequality in (C.9) is from $\mu_{1}>_{M} \mu_{2}$, the second inequality from the individual rationality of $\mu_{2}$, and in both cases the strictness of the inequality 
is from the strictness of preferences. Because of (C.3), an analogous argument yields (C.10). Using these properties, we now argue that the above systems of equations has a solution that satisfies

$$
\begin{aligned}
x^{*}\left(m_{i}, w_{i}\right) & >0, x^{*}\left(m_{i+1}, w_{i}\right)>0, \\
x^{*}\left(m_{i}, w_{i}\right)+x^{*}\left(m_{i+1}, w_{i}\right) & <1, x^{*}\left(m_{i}, w_{i-1}\right)+x\left(m_{1}, w_{i}\right)<1
\end{aligned}
$$

for all $i=1, \cdots, \ell$.

We construct a solution as follows. For any $c \in[0,1]$, let $x\left(m_{1}, w_{\ell}\right)=c$. Using (C.9), the first equation determines $x\left(m_{1}, w_{1}\right) \in[0,1)$ as a strictly decreasing, affine function $h_{1}$ of $c$, which satisfies $h_{1}(1)=0$. Substitute $x\left(m_{1}, w_{1}\right)=h_{1}(c)$ into the next equation, and note that (C.10) determines $x\left(m_{2}, w_{1}\right) \in(0,1)$ as a strictly increasing affine function $h_{2}$ of $c$. Proceeding in this fashion until the last equation is reached, this iterative procedure defines a strictly increasing and affine mapping from $[0,1]$ into $(0,1)$. This function has a unique fixed point $0<c^{*}<1$. Let $x^{*}\left(m_{\ell+1}, w_{\ell}\right)=x^{*}\left(m_{1}, w_{\ell}\right)=c^{*}$ and use $h_{1}, h_{2}, \cdots$ to determine the remaining values. This yields a solution that satisfies (C.11). The inequalities in (C.12) are then implied by (C.9) and (C.10).

Apply this argument to all cycles; and complete the specification of $x^{*}$ by setting $x^{*}(m, w)=0$ for $(m, w)$ satisfying $x_{1}(m, w)=x_{2}(m, w)=0$ and by setting $x^{*}(m, w)=1$ for $(m, w)$ satisfying $x_{1}(m, w)=x_{2}(m, w)=1$, where $x_{1}$ and $x_{2}$ are the assignment matrices corresponding to $\mu_{1}$ and $\mu_{2}$. By construction, $x^{*}$ satisfies (1) - (3) as well as (C.5) and (C.6). Thus, $x^{*}$ is a fractional matching supported by $\mu_{1}$ and $\mu_{2}$. For $m \notin \tilde{M}$ the matching $x^{*}$ satisfies $U\left(m ; x^{*}\right)=u\left(m, \mu_{1}(m)\right)=$ $u\left(m, \mu_{2}(m)\right)$ so that (C.7) is satisfied. Similarly, (C.7) holds for $w \notin \tilde{W}$. For $m \in \tilde{M}$ we have (as $x^{*}$ solves the set of equations given above):

$$
u\left(m, \mu_{2}(m)\right)=x^{*}\left(m, \mu_{1}(m)\right) u\left(m, \mu_{1}(m)\right)+x^{*}\left(m, \mu_{2}(m)\right) u\left(m, \mu_{2}(m)\right) .
$$

As $U\left(m ; x^{*}\right)$ is given by the right side of this equation, (C.7) holds. Similarly, for $w \in \tilde{W}$ we have

$$
\left.V(w ; x)=v\left(\mu_{1}(w), w\right)=x^{*}\left(\mu_{1}(w), w\right) v\left(\mu_{1}(w)\right), w\right)+x^{*}\left(\mu_{2}(w), w\right) v\left(\mu_{2}(w), w\right),
$$

yielding (C.7) for those types.

\section{Appendix D. Proof of Corollary 3}

Let $x_{*}$ be the unique stable matching. By Lemma 9, there exists $\underline{\lambda}_{e}$ such that for all $\lambda>\underline{\lambda}_{e}$ an equilibrium matching $x^{\lambda}$ exists. By Corollary 1 , we have $\lim _{\lambda \rightarrow \infty} x^{\lambda}=x_{*}{ }^{29}$ For $\lambda>\underline{\lambda}_{e}$, let $\alpha^{\lambda}$ denote the acceptance probabilities of the equilibrium inducing $x^{\lambda}$. By Lemma 5, this is well-defined.

Because the payoff functions are continuous, $\lim _{\lambda \rightarrow \infty} U\left(m ; x^{\lambda}\right)=U\left(m ; x_{*}\right)$ and $\lim _{\lambda \rightarrow \infty} V\left(w ; x^{\lambda}\right)=$ $V\left(w ; x_{*}\right)$, for all sequences of equilibrium matchings. Because the number of types is finite, we may therefore choose $\underline{\lambda}_{q} \geq \underline{\lambda}_{e}$ such that for all $\lambda>\underline{\lambda}_{q}$ and all equilibrium matchings $x^{\lambda}$ for $\lambda$,

$$
\begin{gathered}
u(m, w)<U\left(m ; x_{*}\right) \Rightarrow u(m, w)<U\left(m ; x^{\lambda}\right), \\
v(m, w)<V\left(w ; x_{*}\right) \Rightarrow v(m, w)<V\left(w ; x^{\lambda}\right)
\end{gathered}
$$

\footnotetext{
${ }^{29}$ Suppose not. Because the set of matchings is compact, we can then find a sequence $\left(\lambda_{k}\right)$ converging to infinity such that the associated sequence $\left(x_{k}\right)$ of equilibrium matchings with $x_{k}=x^{\lambda_{k}}$ converges to some $\hat{x} \neq x_{*}$. This contradicts Corollary 1 .
} 
hold for all $(m, w)$. We now restrict attention to $\lambda>\underline{\lambda}_{q}$.

Because $x_{*}$ is a simple matching, we have $x_{*}(m, w) \in\{0,1\}$ for all pairs $(m, w)$. Consider any pair $(m, w)$ satisfying $x^{*}(m, w)=0$. Because preferences are strict and $x_{*}$ is stable, this implies either $u(m, w)<U\left(m ; x_{*}\right)$ or $v(m, w)<V\left(w ; x_{*}\right)$, or both. Therefore, from (D.1) - (D.2) and the optimality condition for equilibrium strategies, cf. (23),

$$
x_{*}(m, w)=0 \Rightarrow \alpha^{\lambda}(m, w)=0 .
$$

Consider any pair $(m, w)$ satisfying $x^{*}(m, w)=1$. We then have $U\left(m ; x_{*}\right)=u(m, w)>0$ and $V\left(w ; x_{*}\right)=v(m, w)>0$, where the inequalities are from the individual rationality of $x_{*}$. Because (D.3) implies $x^{\lambda}\left(m, w^{\prime}\right)=x^{\lambda}\left(m^{\prime}, w\right)=0$ for all $m^{\prime} \neq m$ and $w^{\prime} \neq w$ and because $x^{\lambda}(h, h)>0$ holds for all $h$, we have $u(m, w)>U\left(m ; x^{\lambda}\right)$ and $v(m, w)>V\left(w ; x^{\lambda}\right)$. Therefore, the optimality condition for equilibrium strategies implies

$$
x_{*}(m, w)=1 \Rightarrow \alpha^{\lambda}(m, w)=1 .
$$

As $x_{*}(m, w) \in\{0,1\}$ holds for all pairs ( $\left.m, w\right)$, (D.3) - (D.4) imply that the equilibrium acceptance probabilities $\alpha^{\lambda}$ are uniquely determined and satisfy $\alpha^{\lambda}(m, w)=x_{*}(m, w)$ for all $(m, w)$.

Suppose the contact function is given by (31). Using (11) and (31), the conditions characterizing equilibrium matches from Lemma 7 reduce to

$$
x(m, w) \leq \frac{\eta \lambda}{\delta^{2}} x(m, m) x(w, w)
$$

and

$$
x(m, w)= \begin{cases}0 & \text { if } u(m, w)<U(m ; x) \text { or } v(m, w)<V(w ; x), \\ \frac{\lambda \eta}{\delta^{2}} x(m, m) x(w, w) & \text { if } u(m, w)>U(m ; x) \text { and } v(m, w)>V(w ; x) .\end{cases}
$$

From (D.3),

$$
x_{*}(m, w)=0 \Rightarrow x^{\lambda}(m, w)=0 .
$$

We have also shown that for all $(m, w)$ satisfying $x_{*}(m, w)=1$, the inequalities $u(m, w)>$ $U\left(m ; x^{\lambda}\right)$ and $v(m, w)>V\left(w ; x^{\lambda}\right)$ hold. Using (D.6),

$$
x_{*}(m, w)=1 \Rightarrow x^{\lambda}(m, w)=\frac{\lambda \eta}{\delta^{2}} x^{\lambda}(m, m) x^{\lambda}(w, w) .
$$

Because every $m \in M$ and every $w \in W$ has at most one partner in the simple matching $x_{*}$, we can use (D.3) and (D.4) to infer that

$$
x_{*}(m, w)=1 \Rightarrow x^{\lambda}(m, m)=x^{\lambda}(w, w)=1-x^{\lambda}(m, w) .
$$

Consequently, we can rewrite the right side of (D.8) as

$$
x^{\lambda}(m, w)=\frac{\lambda \eta}{\delta^{2}}\left(1-x^{\lambda}(m, w)\right)^{2} .
$$

Equation (D.10) has a unique solution $x^{\lambda}(m, w)$ in the interval [0,1]. Since in every stable simple matching, either $x_{*}(m, w)=0$ or $x_{*}(m, w)=1$, the equilibrium matching $x^{\lambda}(m, w)$ is therefore uniquely determined for all pairs ( $m, w$ ) via (D.7) and (D.8). From Lemma 5 , uniqueness of the equilibrium matching implies uniqueness of equilibrium, finishing the proof. 


\section{References}

[1] H. Adachi, A Search Model of Two-Sided Matching under Nontransferable Utility, Journal of Economic Theory 113 (2003) 182-198.

[2] G. Becker, A Theory of Marriage: Part I, Journal of Political Economy 81 (1973) 813.

[3] F. Bloch, E. Diamantoudi, Noncooperative formation of coalitions in hedonic games, International Journal of Game Theory 40 (2011) 263-280.

[4] F. Bloch, H. Ryder, Two-sided search, marriages, and matchmakers, International Economic Review 41 (2000) 93-116.

[5] E. Budish, Y.K. Che, F. Kojima, P. Milgrom, Designing random allocation mechanisms: Theory and applications, The American Economic Review 103 (2013) 585-623.

[6] K. Burdett, M.G. Coles, Marriage and class, Quarterly Journal of Economics 112 (1997) 141-168.

[7] K. Burdett, M.G. Coles, Long-Term Partnership Formation: Marriage and Employment, The Economic Journal 109 (1999) F307-F334.

[8] K. Burdett, R. Imai, R. Wright, Unstable relationships, Frontiers of Macroeconomics 1 (2004).

[9] K. Burdett, R. Wright, Two-Sided Search with Nontransferable Utility, Review of Economic Dynamics 1 (1998) $220-245$.

[10] S. Clark, The uniqueness of stable matchings, The BE Journal of Theoretical Economics 6 (2006) 8.

[11] P.A. Diamond, E. Maskin, An equilibrium analysis of search and breach of contract I: Steady states, Bell Journal of Economics 10 (1979) 282-316.

[12] F. Echenique, S. Lee, M. Shum, M. Yenmez, The revealed preference theory of stable and extremal stable matchings, Econometrica 81 (2013) 153-171.

[13] J. Eeckhout, Bilateral search and vertical heterogeneity, International Economic Review 40 (1999) 869-887.

[14] J. Eeckhout, On the uniqueness of stable marriage matchings, Economics Letters 69 (2000) 1-8.

[15] D. Gale, Limit theorems for markets with sequential bargaining, Journal of Economic Theory 43 (1987) $20-54$.

[16] D. Gale, Strategic Foundations of General Equilibrium: Dynamic Matching and Bargaining Games, Cambridge University Press, 2000.

[17] D. Gale, L. Shapley, College Admissions and the Stability of Marriage, American Mathematical Monthly 69 (1962) $9-15$.

[18] R. Johnstone, J. Reynolds, J. Deutsch, Mutual Mate Choice and Sex Differences in Choosiness, Evolution 50 (1996) $1382-1391$.

[19] O. Kesten, M.U. Ünver, A Theory of School Choice Lotteries, Technical Report, Boston College, 2013.

[20] S. Lauermann, Dynamic matching and bargaining games: A general approach, American Economic Review 103 (2013) 663-689.

[21] J.M. McNamara, E.J. Collins, The Job Search Problem as an Employer-Candidate Game, Journal of Applied Probability 27 (1990) 815-827.

[22] D. McVitie, L. Wilson, The stable marriage problem, Communications of the ACM 14 (1971) 486-490.

[23] P.B. Morgan, A Model of Search, Coordination and Market Segmentation, Mimeo, Department of Economics, University of Buffalo, 1998.

[24] M.J. Osborne, A. Rubinstein, Bargaining and Markets, Academic Press, Inc., San Diego, CA, 1990.

[25] B. Petrongolo, C. Pissarides, Looking into the black box: A survey of the matching function, Journal of Economic Literature 39 (2001) 390-431.

[26] A. Roth, M.A.O. Sotomayor, Two-Sided Matching: A Study in Game-Theoretic Modeling and Analysis, Econometric Society Monographs, Cambridge University Press, Cambridge, 1990.

[27] A. Roth, J. Vande Vate, Random Paths to Stability in Two-Sided Matching, Econometrica 58 (1990) 1475-1480

[28] A.E. Roth, U.G. Rothblum, J.H. Vande Vate, Stable Matchings, Optimal Assignments, and Linear Programming, Mathematics of Operations Research 18 (1993) 803-828.

[29] U. Rothblum, Characterization of Stable Matchings as Extreme Points of a Polytope, Mathematical Programming 54 (1992) 57-67.

[30] R. Shimer, L. Smith, Assortative matching and search, Econometrica 68 (2000) 343-369.

[31] L. Smith, The marriage model with search frictions, Journal of Political Economy 114 (2006) 1124-1144.

[32] L. Smith, Frictional matching models, Annual Review of Economics 3 (2011) 319-38.

[33] T. Sönmez, Strategy-proofness and essentially single-valued cores, Econometrica 67 (1999) 677-689.

[34] M. Stevens, New microfoundations for the aggregate matching function, International Economic Review 48 (2007) $847-868$.

[35] J.H. Vande Vate, Linear Programming Brings Marital Bliss, Operations Research Letters 8 (1989) 147-153. 